Impulse Response Estimation for Spatial Resolution Enhancement in Ultrasonic NDE Imaging

G. A. Clark

July 7, 2004 
This document was prepared as an account of work sponsored by an agency of the United States Government. Neither the United States Government nor the University of California nor any of their employees, makes any warranty, express or implied, or assumes any legal liability or responsibility for the accuracy, completeness, or usefulness of any information, apparatus, product, or process disclosed, or represents that its use would not infringe privately owned rights. Reference herein to any specific commercial product, process, or service by trade name, trademark, manufacturer, or otherwise, does not necessarily constitute or imply its endorsement, recommendation, or favoring by the United States Government or the University of California. The views and opinions of authors expressed herein do not necessarily state or reflect those of the United States Government or the University of California, and shall not be used for advertising or product endorsement purposes.

This work was performed under the auspices of the U.S. Department of Energy by University of California, Lawrence Livermore National Laboratory under Contract W-7405-Eng-48. 


\title{
Impulse Response Estimation for Spatial Resolution Enhancement in Ultrasonic NDE Imaging
}

\author{
Grace A. Clark, Ph.D. \\ Lawrence Livermore National Laboratory \\ 7000 East Ave., L-130, Livermore, CA 94550 \\ (925) 423-9759 (Office), (925) 422-2495 (FAX), \\ clark9@1lnl.gov
}

June 25, 2004

UCRL-TR-205101 


\section{Acknowledgments}

The author gratefully acknowledges the important contributions to this work made by her colleagues. Steven E. Benson motivated and sponsored this work (about three weeks of my time). Dr. Graham H. Thomas serves as Co-PI on the project. Steve provided ultrasonic measurement data and expert advice. Dr. James V. Candy and Alan W. Meyer provided a MATLAB implementation of the Levinson-Wiggins-Robbins algorithm for inverting a Toeplitz matrix. The Mechanical Engineering Tech Base Program funded the overall project. 


\begin{abstract}
This report describes a signal processing algorithm and MATLAB software for improving spatial resolution in ultrasonic nondestructive evaluation (NDE) imaging of materials. Given a measured reflection signal and an associated reference signal, the algorithm produces an optimal least-squares estimate of the impulse response of the material under test. This estimated impulse response, when used in place of the raw reflection signal, enhances the spatial resolution of the ultrasonic measurements by removing distortion caused by the limited-bandwidth transducers and the materials under test. The theory behind the processing algorithms is briefly presented, while the reader is referred to the bibliography for details. The main focus of the report is to describe how to use the MATLAB software. Two processing examples using actual ultrasonic measurements are provided for tutorial purposes.
\end{abstract}




\section{Contents}

$\begin{array}{lll}1 & \text { Introduction } & 5\end{array}$

1.1 Examples of Ultrasonic Processing Results Using Wiener Impulse Response Estimation and Bandlimited Spectrum Extrapolation $\ldots \ldots \ldots \ldots \ldots \ldots$

1.2 Signals Measured in a Controlled Experiment $\ldots \ldots \ldots \ldots \ldots \ldots$

2 Theoretical Background $\quad 11$

3 Signal Preprocessing $\quad 14$

3.1 Removing Mean and/or Trend from the Signals . . . . . . . . . . . . . . . . . . . . . 14

3.2 Designing the Proper Lengths of the Signals $\ldots \ldots \ldots \ldots \ldots \ldots$

3.3 Cutting a Reference Signal $\ldots \ldots \ldots \ldots \ldots \ldots \ldots \ldots$

3.4 Temporal Shifting/Alignment of the Signals $\ldots \ldots \ldots \ldots \ldots \ldots \ldots$

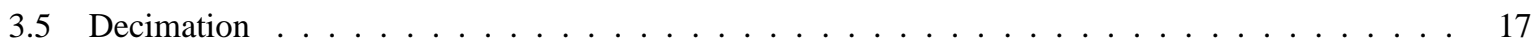

4 Impulse Response Estimation $\quad 26$

5 Signal Post-Processing $\quad 31$

5.1 Including the Artificial Delay of $y(t)$ that was Introduced During Preprocessing . . . . . . . 31

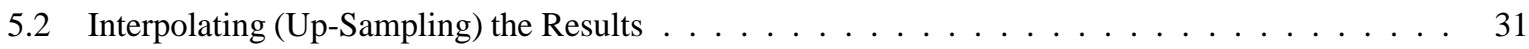

6 Processing Example Using a Back Surface Reflection as the Reference/Input Signal 38

7 Future Work $\quad 44$

8 Conclusions $\quad 45$

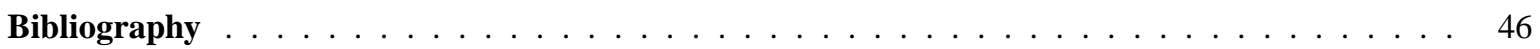




\section{List of Figures}

1.1 Experiment to measure layer thicknesses in a material made of a block of lucite on a block of aluminum. The ideal impulse response of the material consists of three delayed impulses, the first two having negative sign and the last having positive sign. The raw measured ultrasonic waveform $y(t)$ is distorted due to the bandpass nature of the ultrasonic transducer. The estimated impulse response $\widehat{h}(t)$ is a much closer approximation to the ideal impulse response. After bandlimited spectrum extrapolation, the signal $\widehat{h}_{e}(t)$ is a very close approximation to the ideal impulse response. The processed signals are much easier to interpret than the raw data, allowing much improved estimation of travel times and material thicknesses. . . . . . . . . . . . . . . . .

1.2 Controlled Experiment to test ultrasonic methods of crack sizing. The aluminum specimen has a flaw, the location and geometry of which are known. The "crack" was simulated by a saw cut. The reference signal was chosen to be the reflection from a corner, because that is reasonably related to the signal expected from the crack. . . . . . . . . . . . . . .

1.3 Experiment to test ultrasonic methods of crack sizing (see the last figure). (a) The scattered measurement $y(t)$, (b) The estimated impulse response $\widehat{h}(t)$ from the Wiener algorithm, and (c) The extrapolated impulse response $\widehat{h}_{e}(t)$. Note the dramatic improvement seen in $\widehat{h}(t)$ and $\widehat{h}_{e}(t)$. The extrapolated impulse response $\widehat{h}_{e}(t)$ closely approximates the impulse response predicted by theory.

1.4 Experiment to measure/estimate the thickness of the adhesive material deposited between two pieces of aluminum. The ultrasonic data were collected at a part location devoid of significant

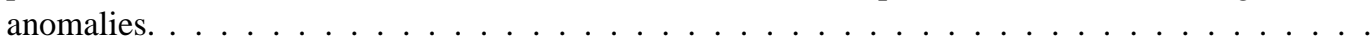

1.5 Experiment to measure/estimate the thickness of the adhesive material deposited between two pieces of aluminum (see the previous figure). The reference signal and measurement signal are used by the Wiener algorithm to estimate the impulse response $\widehat{h}(t)$ of the part. The estimated impulse response is then processed by the bandlimited spectrum extrapolation algorithm to produce the extrapolated impulse response $\widehat{h}_{e}(t)$, which has greatly enhanced spatial resolution. The pulses corresponding to the bond boundaries are not resolvable in the raw measurement, but they are clearly resolvable in the extrapolated impulse response. From the raw measurement, thickness measurements are not feasible. From the processed measurement, the thickness measurements are

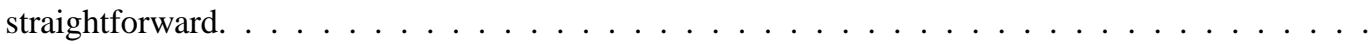

2.1 The System Block Diagram shows a linear system with impulse response $h(t)$ being modeled by impulse response $\widehat{h}(t)$. Here, we let $t$ denote the continuous time variable $\ldots \ldots \ldots \ldots$

3.1 The raw reference signal $x(t)$ and measurement signal $y(t)$ are plotted for comparison. $\ldots \ldots .15$ 
3.2 The reference signal $x(t)$ and the measurement signal $y(t)$ after mean and trend removal. As one can see, the data acquisition system is so well calibrated that mean/trend removal is not really

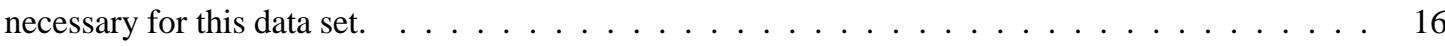

3.3 The cut and post-padded reference signal $x(t) \ldots \ldots \ldots \ldots \ldots$

3.4 The cut and post-padded reference signal $x(t)$ plotted over the measurement $y(t)$ for delay/alignment

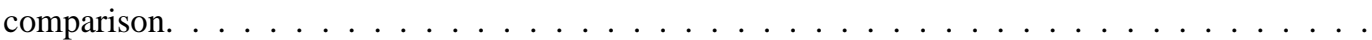

3.5 Reference signal $x(t)$, output measurement $y(t)$ and the delayed measurement $y(t-\Delta)$ plotted

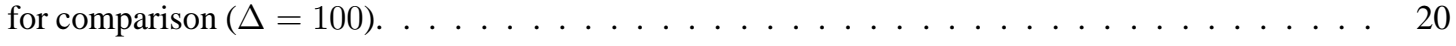

3.6 Reference $x(t)$, plotted over the delayed measurement $y(t-\Delta)$ for comparison. . . . . . . . 21

3.7 $|X(f)|^{2}$ and $|Y(f)|^{2}$ before decimation. The sampling frequency is $f_{s}=10 . e 7 \mathrm{~Hz}$, so the folding frequency is $f_{s} / 2=5 . e 7 \mathrm{~Hz}$. Note that due to oversampling, the number of spectral zeros is very

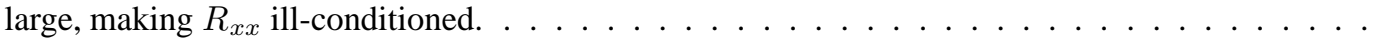

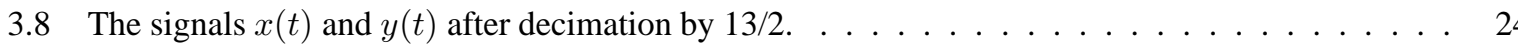

$3.9|X(f)|^{2}$ and $|Y(f)|^{2}$ after decimation by $13 / 2$. Note that the high frequency spectral zeros have been effectively eliminated by the decimation step. . . . . . . . . . . . . . .

4.1 The optimal least squares Wiener estimate of the impulse response $\widehat{h}(t) . N=197, \alpha=5.0$, Range of $\alpha$ is .0182 to 8.7912 . Note: we can now see clearly that, as predicted by theory, the polarity of the front reflection is positive, the polarity of the hole reflection is negative, and the polarity of the back reflection is negative. . . . . . . . . . . . . . . . . .

4.2 The estimated output $\widehat{y}(t)$ is plotted over the measured output $y(t)$. Also shown is the error signal $e(t)=y(t)-\widehat{y}(t)$. Notice that the magnitude scale for the error is very small, and that the error is very small, even in the regions of maximum error. . . . . . . . . . . . . . .

4.3 The estimated output $\widehat{y}(t)$ is plotted along with the estimated impulse response $\widehat{h}(t)$ and the error

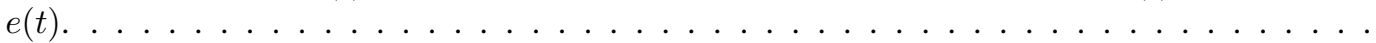

4.4 The input $x(t)$, output $y(t)$, estimated output $\widehat{y}(t)$ and the estimated impulse response $\widehat{h}(t)$ are plotted for comparison. Note that $\widehat{h}(t)$ does not align temporally with $y(t) \ldots \ldots \ldots \ldots$

5.1 The estimated measured output $y(t)$, the estimated impulse response $\widehat{h}(t)$ and the delayed estimated impulse response $\widehat{h}(t-\Delta)$ are plotted for comparison. Notice that once the delay $\Delta$ is included, the signals $y(t)$ and $\widehat{h}(t-\Delta)$ align nicely. This effect is seen more easily in the next figure. 32

5.2 The estimated delayed impulse response $\widehat{h}(t-\Delta)$ is plotted over the measured output $y(t)$ for comparison. Notice that once the delay $\Delta$ is included, the signals $y(t)$ and $\widehat{h}(t-\Delta)$ align nicely.

5.3 The magnitude squared of the DFT's of $\widehat{y}(t)$ and $\widehat{h}(t),|\widehat{Y}(f)|^{2}$ and $|\widehat{H}(f)|^{2}$ before interpolation. . 34

$5.4 \widehat{y}(t)$ and $\widehat{h}(t)$ after interpolation. For some users, this display may be more pleasing to the eye than the decimated version shown earlier. . . . . . . . . . . . . . . .

5.5 The magnitude squared of the DFT's of $\widehat{y}(t)$ and $\widehat{h}(t),|\widehat{Y}(f)|^{2}$ and $|\widehat{H}(f)|^{2}$ after interpolation by

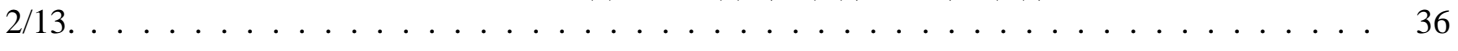

$5.6 \widehat{h}(t)$ plotted over $\widehat{y}(t)$ after post-processing, including interpolation. . . . . . . . . . 37 
6.1 The cut and post-padded back surface reference signal $x(t)$. Note that the sign of the original back surface wavelet has been inverted in the reference signal. . . . . . . . . . . . . . . 39

6.2 Back Surface Reference Case - The signals $x(t)$ and $y(t)$ after decimation by $13 / 2 \ldots \ldots$

6.3 Back Surface Reference Case - $|X(f)|^{2}$ and $|Y(f)|^{2}$ after decimation by 13/2. Note that the high frequency spectral zeros have been effectively eliminated by the decimation step. . . . . . . . . .

6.4 Back Surface Reference Case - The optimal least squares Wiener estimate of the impulse response $\widehat{h}(t) . N=197, \alpha=6.7885$, Range of $\alpha$ is .0001 to 6.7885 . Note: we see that the front reflection is more distorted relative to the case in which we used a front surface reflection as the reference signal. This is, of course, expected, because the back reflection does not match the front reflection. The polarity of the hole reflection is now clearly negative, as we expect from theory. In addition, the hole reflection wavelet now has more of a "spike" "shape as desired. However, the bias in the estimate caused by the regularization is apparent in the low frequency trend introduced into the signal. Fortunately, the bias is probably not enough to be detrimental for our purposes. Also,

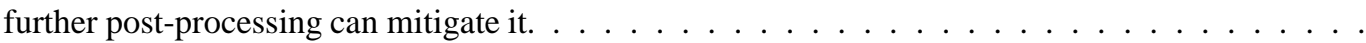

6.5 $\widehat{h}(t)$ plotted over $\widehat{y}(t)$ after post-processing, including interpolation. The bias in the estimate caused by regularization is apparent in the low frequency trend introduced into $\widehat{y}(t)$. Fortunately, the bias is probably not enough to be detrimental for our purposes. Also, further post-processing can mitigate it. . . . . . . . . . . . . . . . . . . . . . . . . 


\section{Chapter 1}

\section{Introduction}

Impulse response estimation for enhancing spatial resolution in ultrasonic NDE imaging has been discussed extensively by the author $[1,2,3,4,5,6,7,8,9,10,11,13,14]$. The cited work was done some years ago, and since then, the software (Fortran) and expertise are no longer available to the NDE Section. The purpose of this work is to reconstitute the expertise and software tools by implementing the appropriate algorithms in MATLAB for use by today's Ultrasonic NDE Section at LLNL. The MATLAB code described in this report is a "research code," meaning that it proves the algorithmic principles for any given single A-scan. Further work will be necessary to write is as a production code that can be used for processing B-scans and blocks of B-scans.

The main purpose of this document is to aid the user in applying the MATLAB software to NDE data. For most of the theoretical development, the reader is referred to the bibliography. Copies of the papers are available from the author.

\subsection{Examples of Ultrasonic Processing Results Using Wiener Impulse Re- sponse Estimation and Bandlimited Spectrum Extrapolation}

The work cited above includes both impulse response esitimation using a Wiener estimator and bandlimited spectrum extrapolation of the estimated impulse response using the method of alternating orthogonal projections to further enhance the spatial resolution of ultrasonic images. Three examples from past research are shown here to introduce the techniques. Explanations are given in the figure captions.

\subsection{Signals Measured in a Controlled Experiment}

In order to test the signal processing algorithms, Steve Benson provided a set of data from a controlled experiment. Holes of known depth, diameter and location were drilled in a block of aluminum. Tape was applied to the back surface to cover the holes and not allow water to enter them. This block was insonified using ultrasonic transducers in a water tank. This report shows processing results for the hole called "H1."

If the situation were ideal, the transducer and the measurement system would have infinite bandwidth so the measured response would show three ideal impulses. The reflection from the front surface would show an ideal positive-going impulse as the waves traversed the water-aluminum interface. The reflection from the hole would give a negative-going impulse corresponding to the waves traversing the aluminum-to-air interface. The back reflection from the tape would give a negative-going pulse as the waves traversed the tape-to-water interface. 

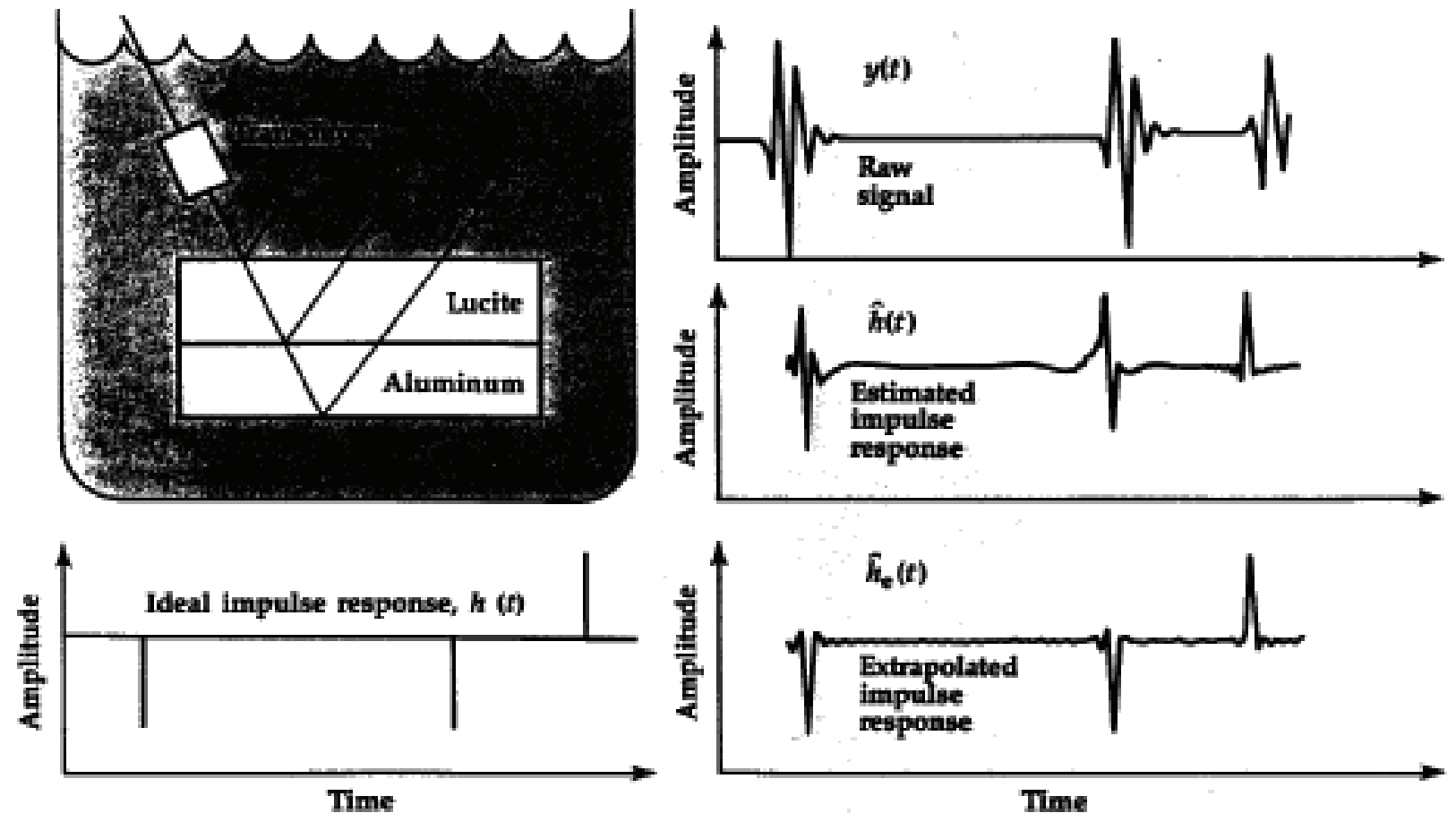

Figure 1.1: Experiment to measure layer thicknesses in a material made of a block of lucite on a block of aluminum. The ideal impulse response of the material consists of three delayed impulses, the first two having negative sign and the last having positive sign. The raw measured ultrasonic waveform $y(t)$ is distorted due to the bandpass nature of the ultrasonic transducer. The estimated impulse response $\widehat{h}(t)$ is a much closer approximation to the ideal impulse response. After bandlimited spectrum extrapolation, the signal $\widehat{h}_{e}(t)$ is a very close approximation to the ideal impulse response. The processed signals are much easier to interpret than the raw data, allowing much improved estimation of travel times and material thicknesses. 


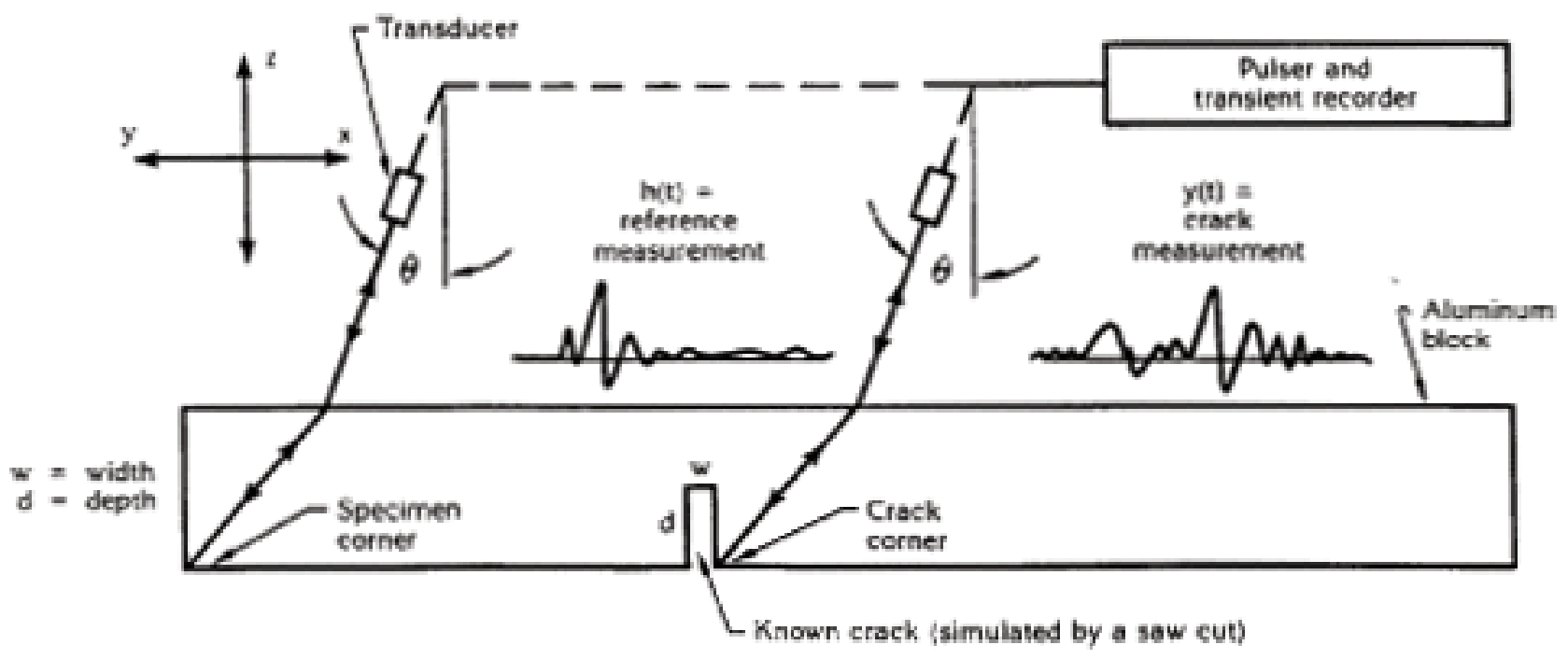

Figure 1.2: Controlled Experiment to test ultrasonic methods of crack sizing. The aluminum specimen has a flaw, the location and geometry of which are known. The "crack" was simulated by a saw cut. The reference signal was chosen to be the reflection from a corner, because that is reasonably related to the signal expected from the crack.

Of course, in practical measurements, the transducers and measurement system have limited bandwidth, so the reflections are not ideal and contain considerable ringing. The data discussed here are very clean with very high signal-to-noise ratio. Even so, due to distortion (ringing), the author is unable to identify conclusively the polarities of the two small reflections. 

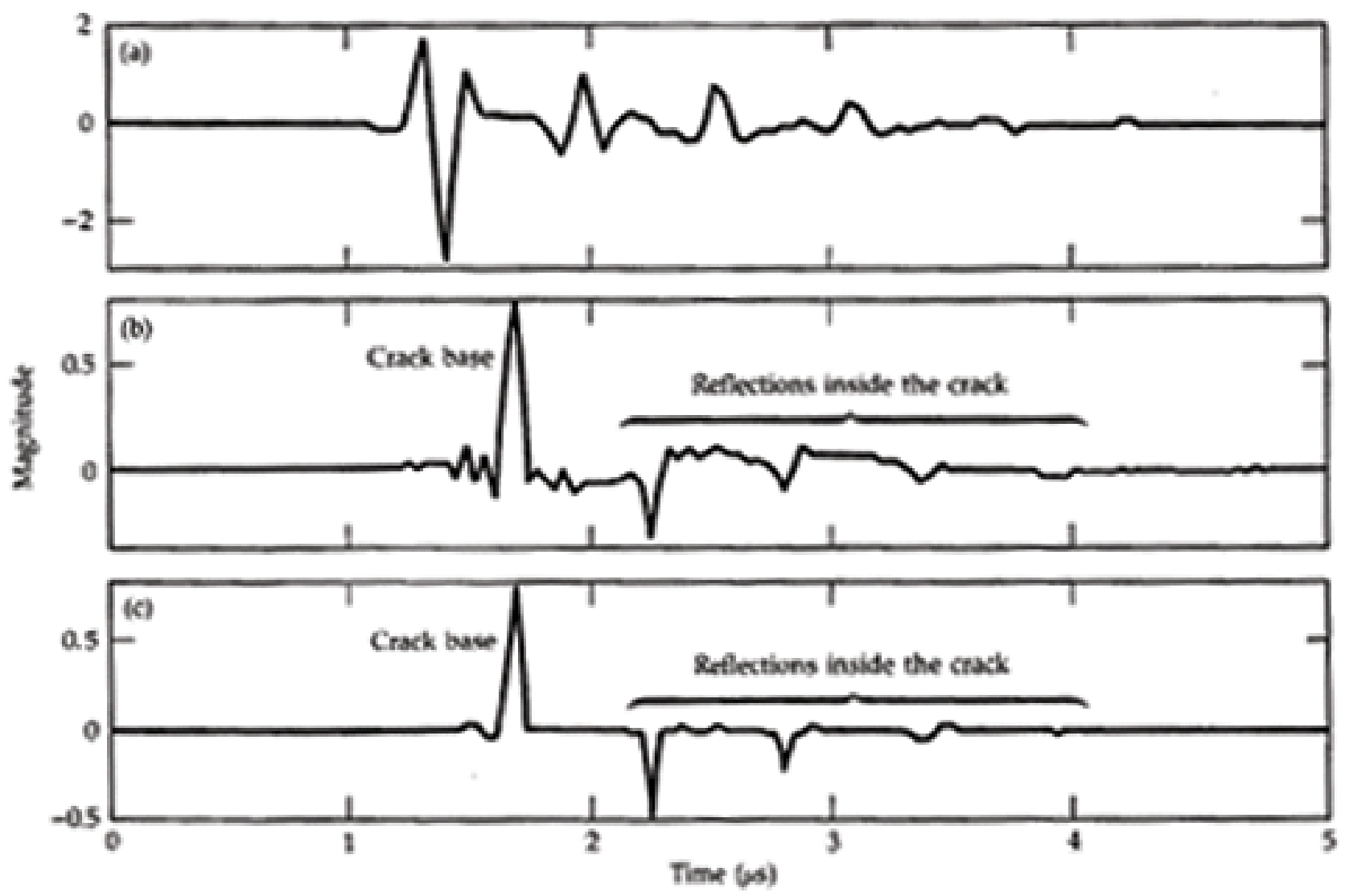

Figure 1.3: Experiment to test ultrasonic methods of crack sizing (see the last figure). (a) The scattered measurement $y(t)$, (b) The estimated impulse response $\widehat{h}(t)$ from the Wiener algorithm, and (c) The extrapolated impulse response $\widehat{h}_{e}(t)$. Note the dramatic improvement seen in $\widehat{h}(t)$ and $\widehat{h}_{e}(t)$. The extrapolated impulse response $\widehat{h}_{e}(t)$ closely approximates the impulse response predicted by theory. 


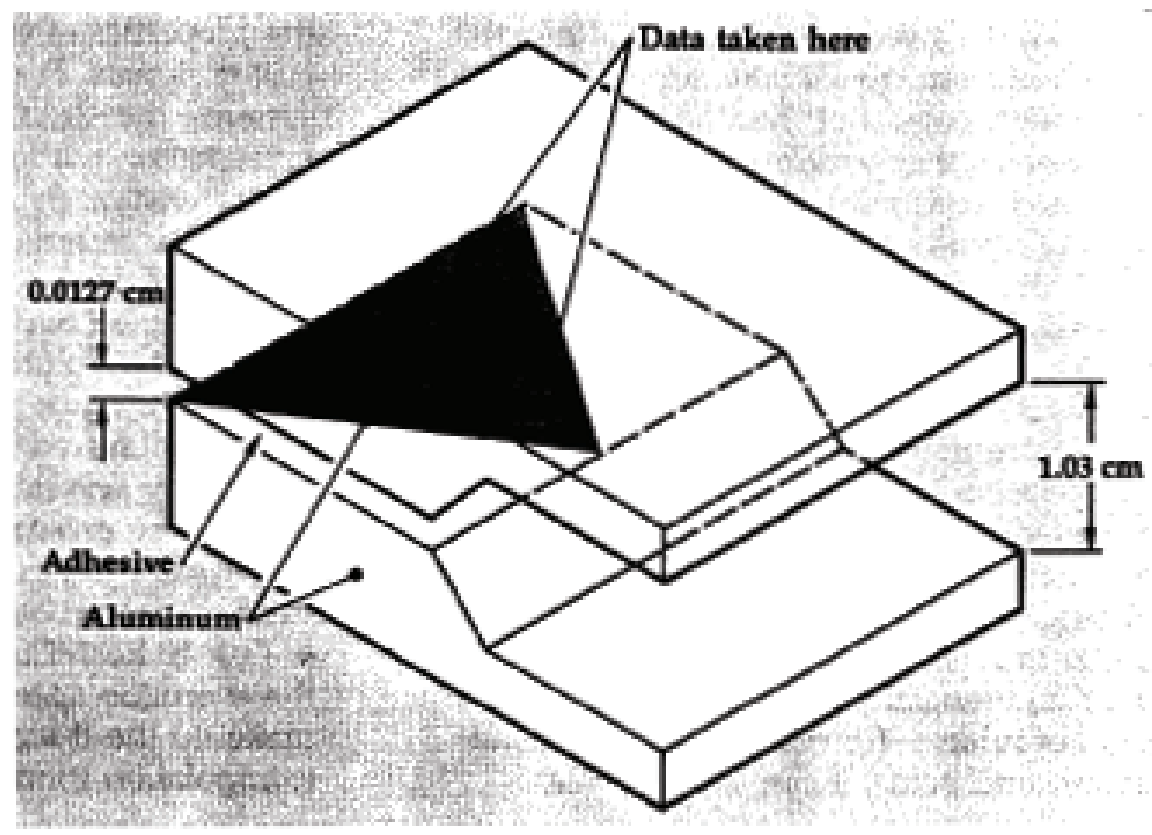

Figure 1.4: Experiment to measure/estimate the thickness of the adhesive material deposited between two pieces of aluminum. The ultrasonic data were collected at a part location devoid of significant anomalies. 


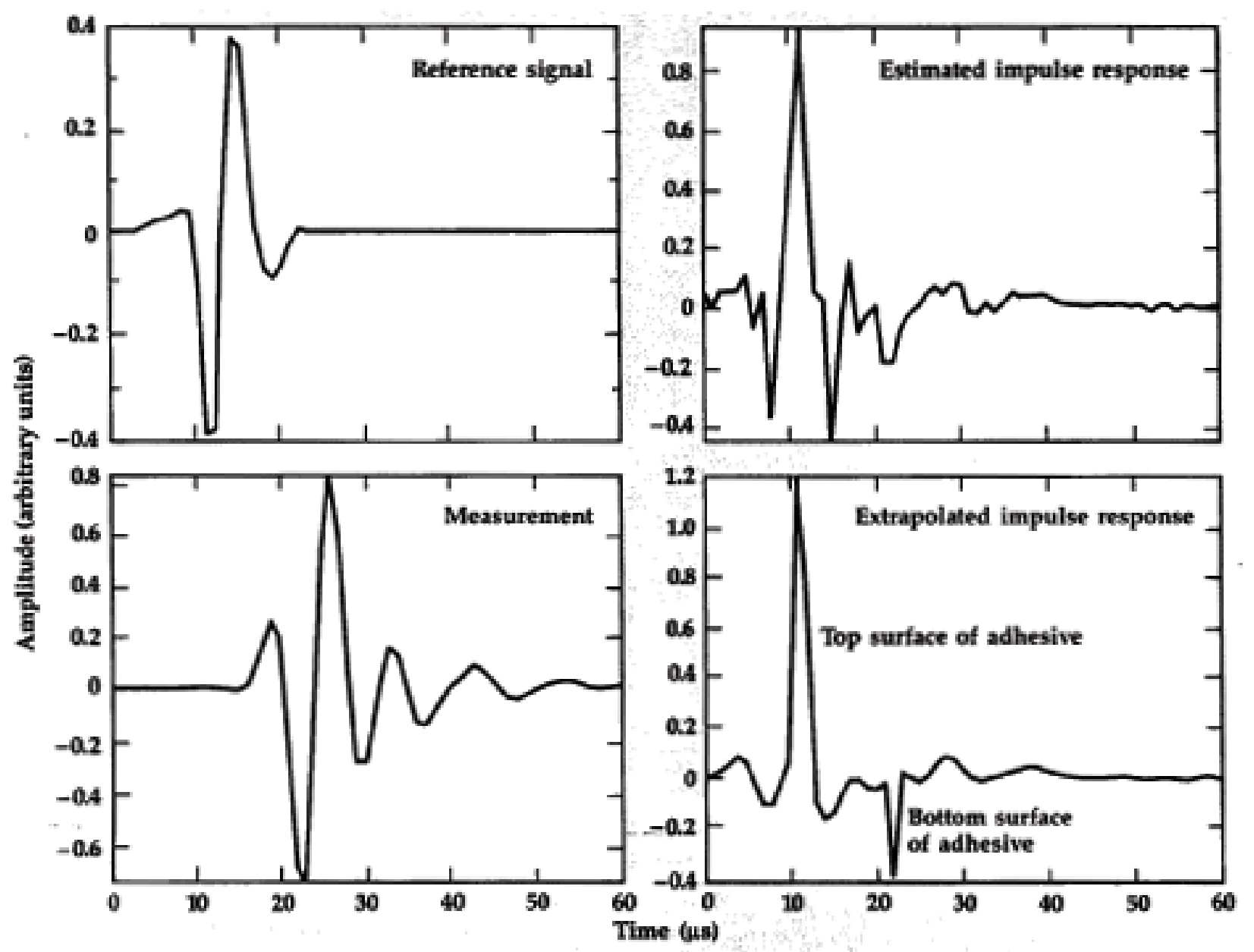

Figure 1.5: Experiment to measure/estimate the thickness of the adhesive material deposited between two pieces of aluminum (see the previous figure). The reference signal and measurement signal are used by the Wiener algorithm to estimate the impulse response $\widehat{h}(t)$ of the part. The estimated impulse response is then processed by the bandlimited spectrum extrapolation algorithm to produce the extrapolated impulse response $\widehat{h}_{e}(t)$, which has greatly enhanced spatial resolution. The pulses corresponding to the bond boundaries are not resolvable in the raw measurement, but they are clearly resolvable in the extrapolated impulse response. From the raw measurement, thickness measurements are not feasible. From the processed measurement, the thickness measurements are straightforward. 


\section{Chapter 2}

\section{Theoretical Background}

The mathematical problem we are solving is known as the system identification problem; Given a linear time invariant system for which we know the inputs $x(n)$ and outputs $y(n)$, estimate the impulse response $h(n)$ of the system $[22,25,20,6]$. Let $n$ denote the discrete time index. We have chosen to use a finite impulse response (FIR), (or all-zero, or moving average) model having $\mathrm{N}$ coefficients we wish to estimate.

Early work in impulse response estimation includes [17, 18]. An excellent presentation of the LevinsonWiggins-Robbins algorithm for efficient inversion of Toeplitz matrices appears in [21]. In fact, this book documents the theory behind J. V. Candy's MATLAB function "lwr "for implementing the algorithm. The author's code Wiener GAC calls the lwr algorithm as part of the computation of the Wiener solution.

A block diagram depicting the mathematical problem to be solved in the system under test is given in Figure (2.1) [1].

The model can be expressed as a convolution:

$$
\widehat{y}(n)=\sum_{k=0}^{N-1} h(k) x(n-k)
$$

The error between the measured response and the estimated response is then

$$
e(n)=y(n)-\widehat{y}(n)
$$

Assuming the measurements are ergodic [24], we wish to perform a least-squares optimization problem using as performance criterion the sum squared error

$$
E=\sum_{n} e(n)
$$

The goal then, is to estimate the $h(n)$ which minimize $E$. The range of the summation index in the definition of $E$ is not specified, but it can be considered to run from minus infinity to infinity. The measurements $x(n)$ and $y(n)$ have finite durations. However, we can consider that they have extensions (via zero-padding) over an infinite range.

From estimation theory, we know that for the minimum squared error solution, the error sequence must be orthogonal to the data $x(n)$ (the orthogonality principle). With some manipulation, we can show that this can be expressed by the Wiener-Hopf equation:

$$
R_{x x} \underline{h}=R_{y x}
$$




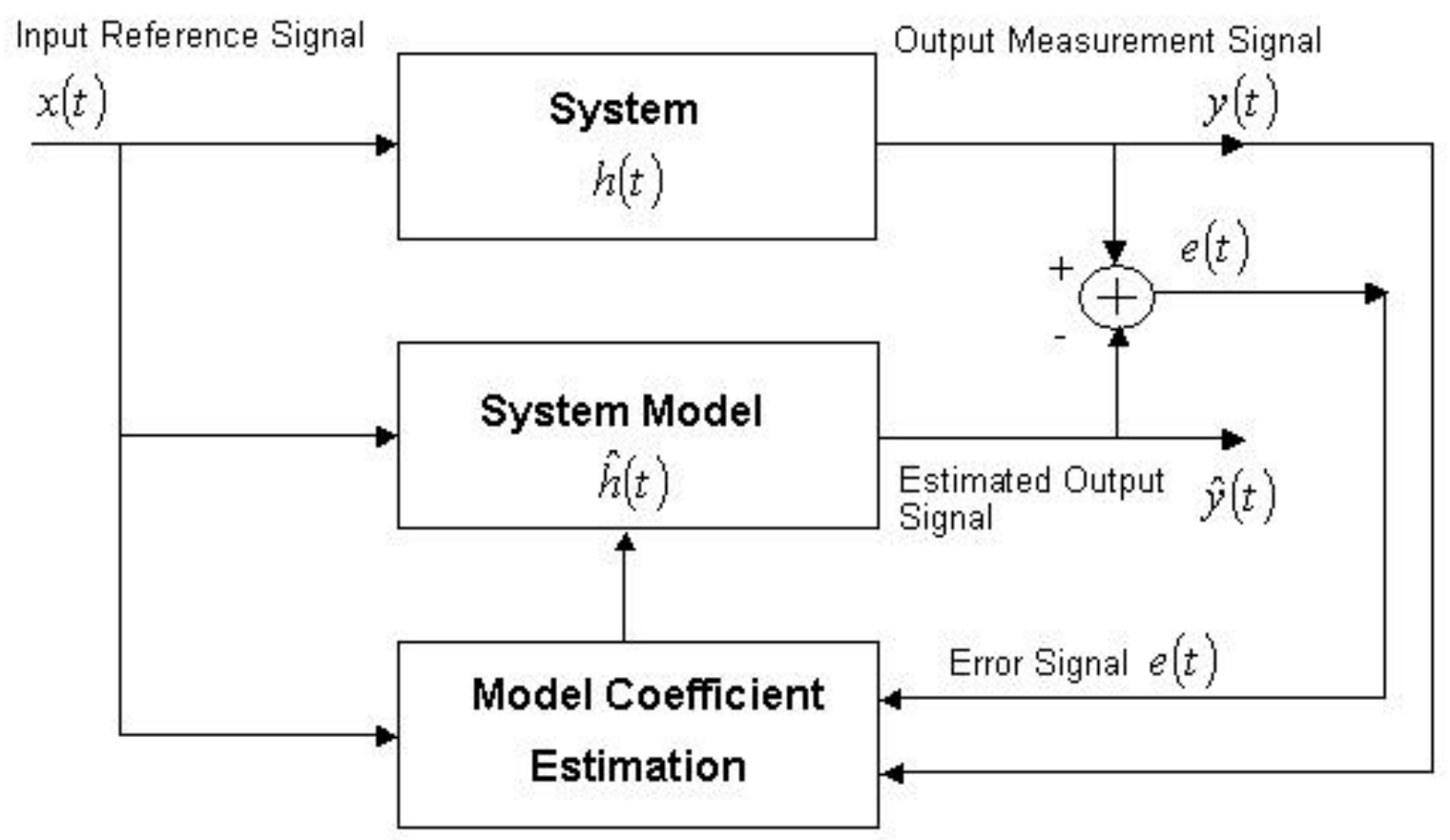

Figure 2.1: The System Block Diagram shows a linear system with impulse response $h(t)$ being modeled by impulse response $\widehat{h}(t)$. Here, we let t denote the continuous time variable 
where $R_{x x}$ is the $N X N$ input autocorreleation matrix, $\underline{h}$ is the $N X 1$ vector of impulse response coefficients, and $R_{y x}$ is the $N X 1$ cross-correlation matrix between the output $y(n)$ and the input $x(n)[1,21,22,23,24,25]$.

The solution of the Wiener-Hopf equation requires the inversion of the Toeplitz input autocorrelation matrix $R_{x x}$.

$$
\underline{h}^{*}=R_{x x}^{-1} R_{y x}
$$

where the asterisk denotes the optimal least squares (Wiener) solution for $h$. One of the properties of the Toeplitz matrix $R_{x x}$ is that its eigenvalues are asymptotically distributed as equally spaced of the spectrum of $x(n)$ [19]. The condition number of $R_{x x}$ is roughly determined by the ratio of the largest to the smallest eigenvalues of the matrix [1]. This means that the condition number is also approximately the ratio of the largest to the smallest values of the spectrum of $x(n)$. The larger the condition number, the more ill-conditioned the matrix is, and the more difficult it is to invert the matrix [31]. The key point is that if there are zeros in the spectrum of $x(n)$, then the condition number is large, the set of equations (inversion of the Toeplitz matrix) is ill-conditioned, and the solutions calculated are not of good quality. Therefore, anything we can do to reduce the number of digital spectral zeros will help us achieve a better solution.

There are two main methods for reducing the number of digital spectral zeros. The First is to decimate the data so that the digital spectrum contains a minimum of high-frequency zeros. We discuss this in the next section. $\underline{\text { Second }}$, we can regularize the ill-posed inversion problem [1] by adding a small positive regularization constant $\alpha$ to the diagonal elements of $R_{x x}$ to shift any small eigenvalues away from zero. In the frequency domain, this is equivalent to adding a small positive constant to the input Power Spectral Density function $S_{x x}(f)$ [1]. This has the effect of raising the spectral floor in the denominator of the Wiener solution to avoid spectral zeros.

The overall effect of the reqularization technique is to reduce estimation error variance at the expense of producing a biased estimate $[1,22,23,24]$. This error variance vs. bias tradeoff is important to consider when choosing the regularization constant $\alpha$. One must choose $\alpha$ large enough to move the eigenvalues of $R_{x x}$ away from zero, but not so large that the bias in the estimate of the impulse response is intolerable.

In the MATLAB software, the user can obtain the minimum and maximum reasonable values of $\alpha$ using the Singular Value Decomposition (SVD) of $R_{x x}$. The user is given an upper and lower bound on the values of $\alpha$ from which to choose. The user can then run the code several times, trying various values of $\alpha$ until a satisfactory result is obtained. Fortunately, the solutions are not very sensitive to the values of $\alpha$, so finding a satisfactory value is not difficult. A rule of thumb is: If the signal is not very noisy, the value of $\alpha$ can be very small. The noisier the data, the larger $\alpha$ needs to be.

Note that if we introduce a delay $\Delta$ to align $x(n)$ and $y(n)$ as discussed in the next section, this corresponds to replacing $y(n)$ with $y(n-\Delta)$ in the formulation given above and proceeding as before. 


\section{Chapter 3}

\section{Signal Preprocessing}

Signal preprocessing is extremely important to the practical success of the impulse response estimation algorithm. The preprocessing steps ensure that the the signals satisfy the algorithm assumptions and give the algorithm the best chance to overcome the fact that the basic mathematical problem is ill-posed [1]. In addition, the preprocessing steps allow us to put the data in a form that is most easily interpreted. The MATLAB script (an M-file) that does the preprocessing is called "Pre Wiener GAC".

Figure (3.1) shows the raw reference signal $x(t)$ and the raw measurement signal $y(t)$. The reference signal is a front and back reflection from a piece of aluminum (with no hole, etc.). The $y(t)$ signal is for an aluminum block containing a hole called "H1".

\subsection{Removing Mean and/or Trend from the Signals}

Removing any statistical mean and/or trend from the data is done to help satisfy the linearity assumption for the system under test. A system with any form of bias (such as a mean and/or trend in the measurements) cannot, by definition, be linear [26, 27]. Since the Wiener estimation algorithm assumes linearity, it is very important to execute this step. Generally, any bias in the measurements is an artifact of the measurement process, and should be removed in any case. Fortunately, for the ultrasonic NDE data acquired in the NDE lab, there is generally little or no mean or trend because the measurement system has been well-designed and calibrated.

Figure (3.2) shows the reference signal $x(t)$ and the measurement signal $y(t)$ after mean and trend removal.

\subsection{Designing the Proper Lengths of the Signals}

Letting $n$ denote the discrete time index, we know from linear system theory that if we convolve a sequence $x(n)$ with a sequence $h(n)$ to obtain a sequence $y(n)$, the following is true: If the length of $x(n)$ is $N_{x}$ samples and the length of $h(n)$ is $N_{h}$ samples, then the length of $y(n)$ will be $N_{x}+N_{h}-1$ samples [27]. In practice, we wish to be able to display an impulse response $h(n)$ that has the same length as the measurement $y(n)$. Clearly, we must carefully account for all of these effects in the design of our signal lengths.

If the length of the nonzero pulse in the reference signal is $N_{x p}$ and the length of a reflection pulse in the impulse response is $N_{h p}$, then the length of the resulting pulse in the measurement signal is $N_{y p}$.

So, the way we design the signal lengths for our practical data set is as follows: First, we know that the length of $y(n)$ is 1280 samples at $f_{s}=1 . e 8 H z$. From the figure we see that we have left an ample number of background 

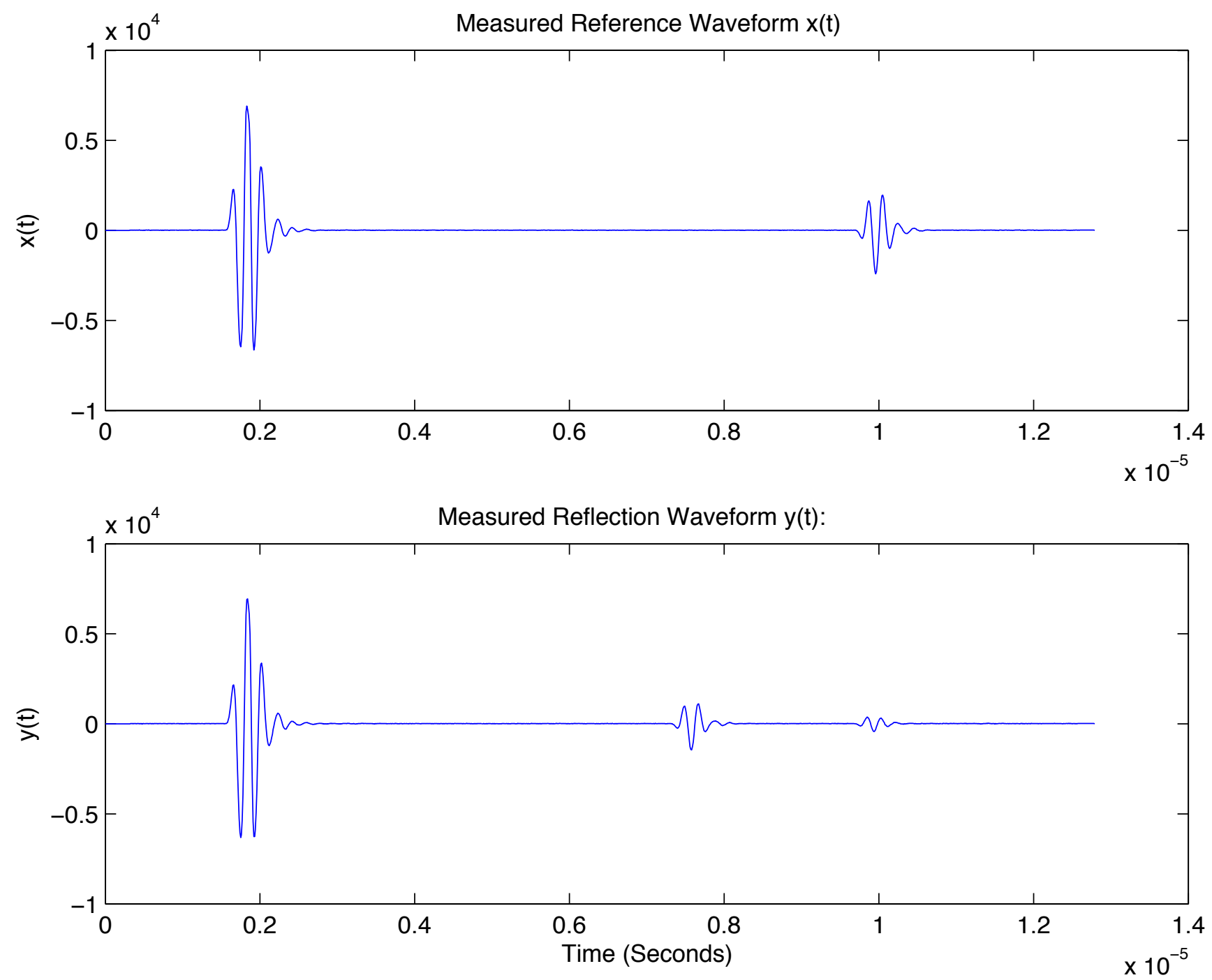

Figure 3.1: The raw reference signal $x(t)$ and measurement signal $y(t)$ are plotted for comparison. 

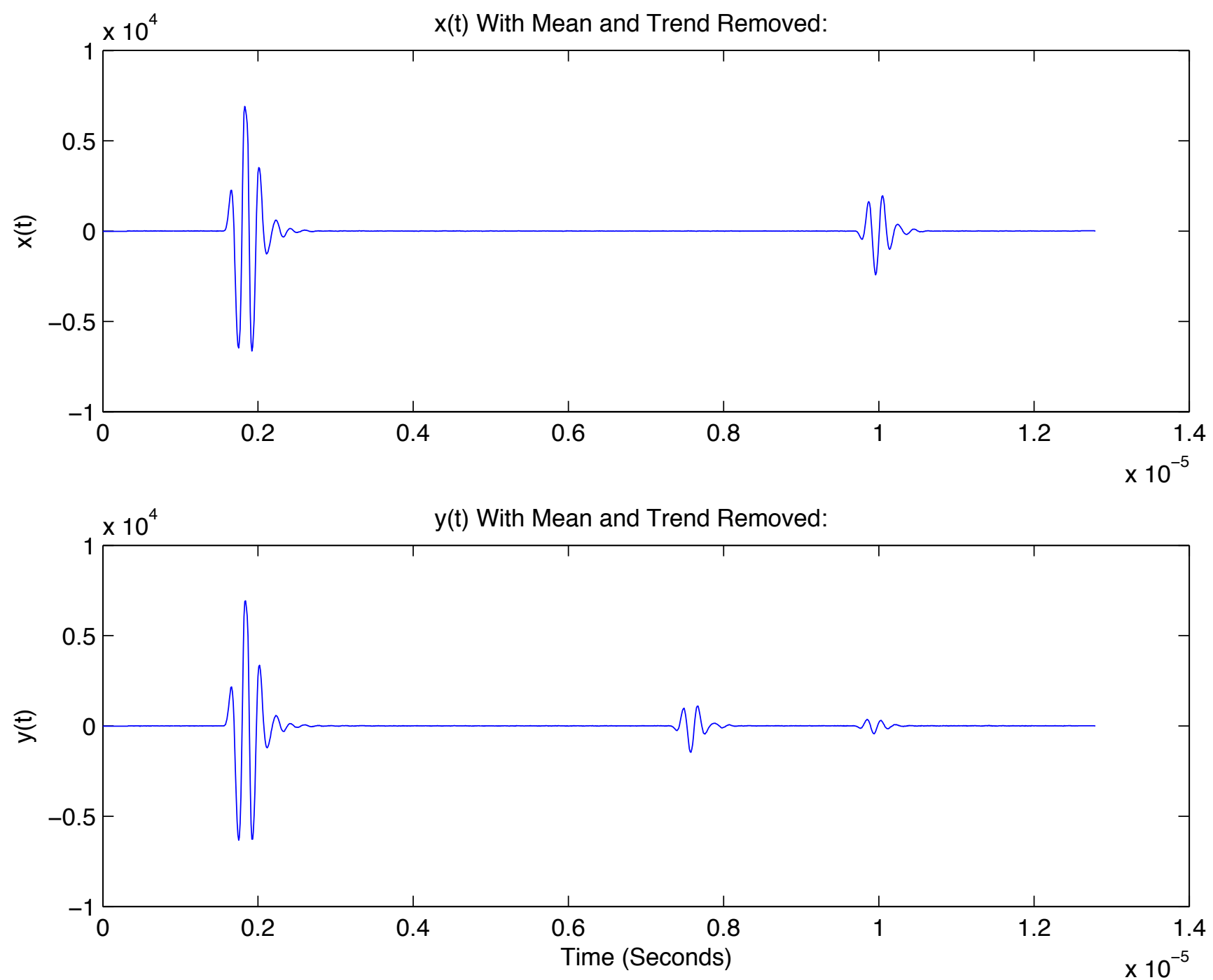

Figure 3.2: The reference signal $x(t)$ and the measurement signal $y(t)$ after mean and trend removal. As one can see, the data acquisition system is so well calibrated that mean/trend removal is not really necessary for this data set. 
samples before and after the pulses of interest. Second, When we cut the reference signal as in the next section, we want to zero-pad the reference pulse so the length of $x(n)$ is the same as the length of $y(n)$. Third, We can let the length of $h(n)$ equal the length of $y(n)$ as long as $N_{x p}+N_{h p}-1$ is short enough so that the convolved pulse fits into the 1280 samples. In our case, it does; so our problem is simple.

\subsection{Cutting a Reference Signal}

A key component of the impulse estimation approach is providing a good quality reference signal [1]. The most common way to produce such a reference is to take a reflection measurement from the front or back surface of the part under test or a piece of material that has the same material properties as the part under test. Sometimes a corner reflection or other specialized reference signal can be used [1]. The MATLAB code "Pre Wiener GAC "gives the user the ability to cut out a piece of a given waveform, and zero-pad it on both ends for use as a reference signal. The user must provide a set of parameters such as time at which to start cutting, number of seconds of data to save after cutting, desired length of the reference signal, etc.

Figure (3.3) shows the cut and post-padded reference signal $x(t)$.

Figure (3.4) shows the cut and post-padded reference signal $x(t)$ plotted over the measurement $y(t)$ for delay/alignment comparison.

\subsection{Temporal Shifting/Alignment of the Signals}

For most measurements, it is necessary to adjust the delay between the reference signal $x(t)$ and the measurement signal $y(t)$. This shifting/aligning of the signals allows the results to be displayed in a form that is easily interpretable by the user [1]. For the data set used here, we offset $x(n)$ and $y(n)$ by 100 samples $(\Delta=100)$.

Figure (3.5) shows the Reference $x(t)$, measurement $y(t)$ and the delayed measurement $y(t-\Delta)$ plotted for comparison.

Figure (3.6) shows the Reference $x(t)$, plotted over the delayed measurement $y(t-\Delta)$ for comparison $(\Delta=$ 100).

\subsection{Decimation}

To decimate a signal is to reduce its sampling frequency $f_{s}$. To interpolate a signal is to increase its sampling frequency. When taken together, Decimation and Interpolation are commonly called Resampling [1, 2, 15, 16, 28]. The author has published in the area of resampling signals and the importance of resampling to the impulse estimation problem $[2,15,16]$. The signals measured in the NDE lab are usually oversampled, in the sense that the sampling frequency is much larger than twice the bandwidth of the signal spectrum (the Nyquist frequency). This is good practice, because it ensures that the data are not aliased [27]. However, for impulse response estimation, it has a very negative effect that can be remedied easily by decimating the data to reduce the available digital frequency bandwidth so it approximates the support of the spectrum of $x(t)$. We now discuss why this is good practice.

For our data set, the sampling frequency is $f_{s}=10 . e 7 \mathrm{~Hz}$, so the folding frequency is $f_{s} / 2=5 . e 7 \mathrm{~Hz}$. The bandwidth is approximately $.8 \mathrm{e} 7 \mathrm{~Hz}$. Note that due to oversampling, the number of spectral zeros is very large, making $R_{x x}$ ill-conditioned. Decimation can remove the high-frequency zeros, but the low frequency zeros and any in-band zeros must be dealt with in other ways. Methods include regularization (as described in the theory 


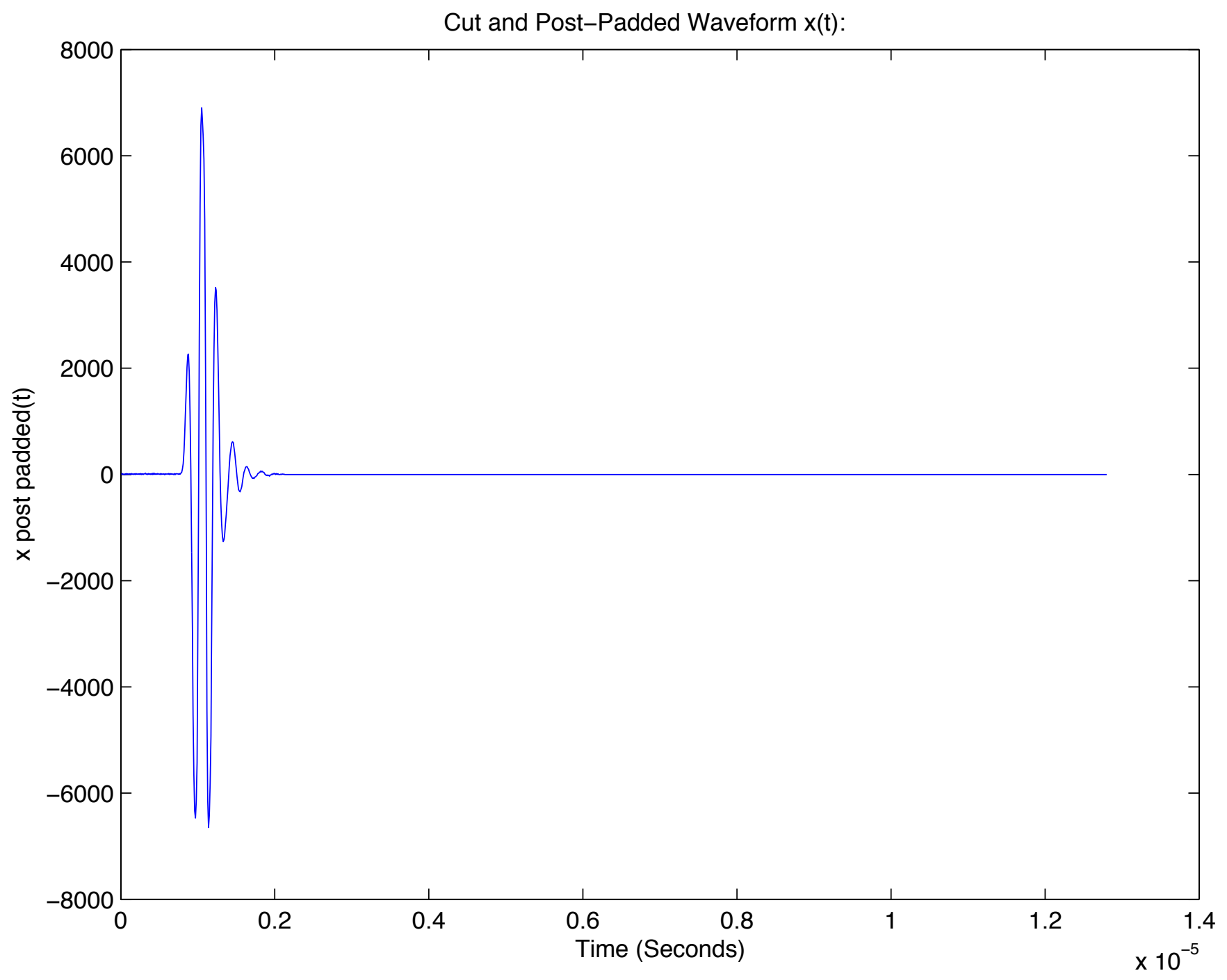

Figure 3.3: The cut and post-padded reference signal $x(t)$. 


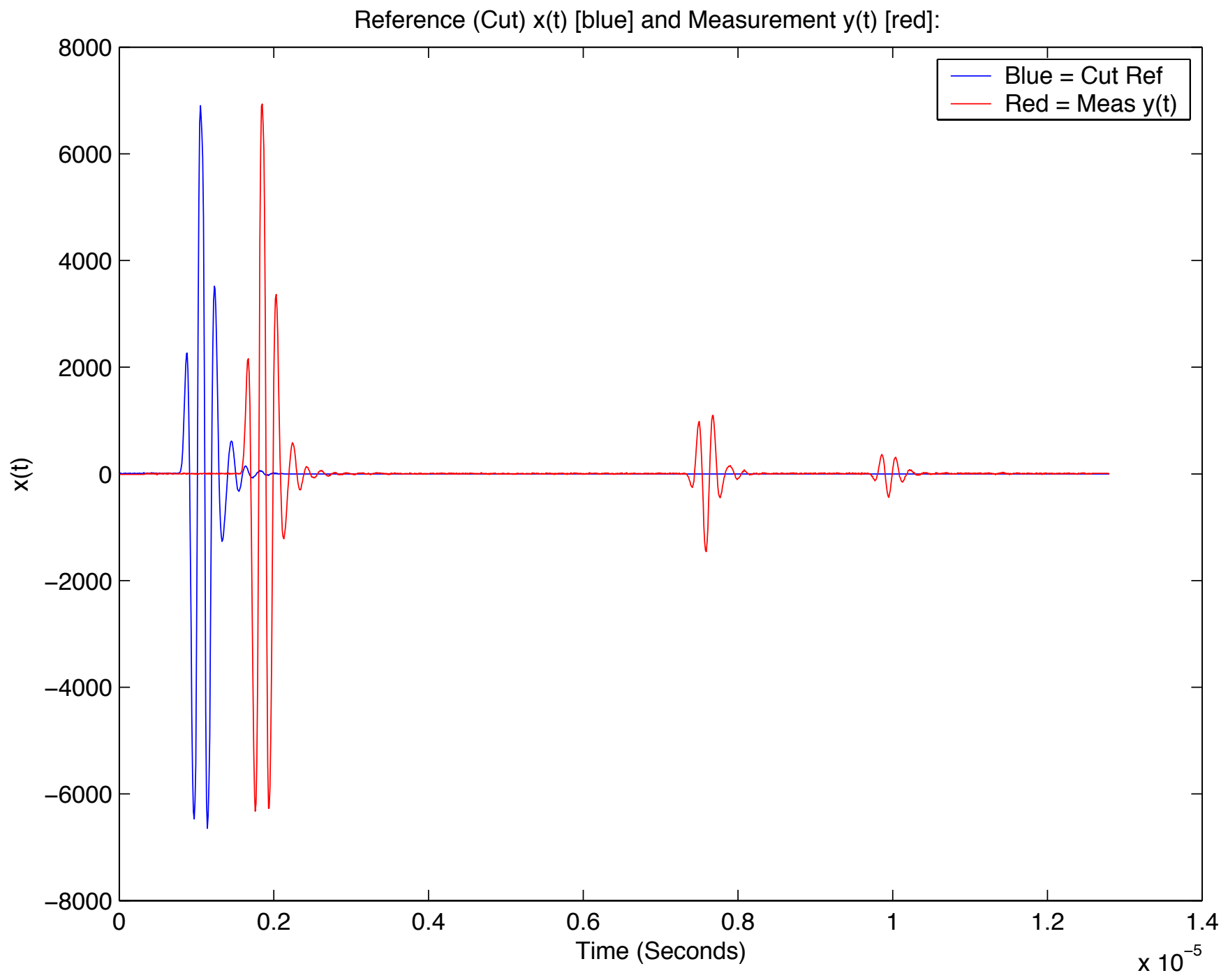

Figure 3.4: The cut and post-padded reference signal $x(t)$ plotted over the measurement $y(t)$ for delay/alignment comparison. 

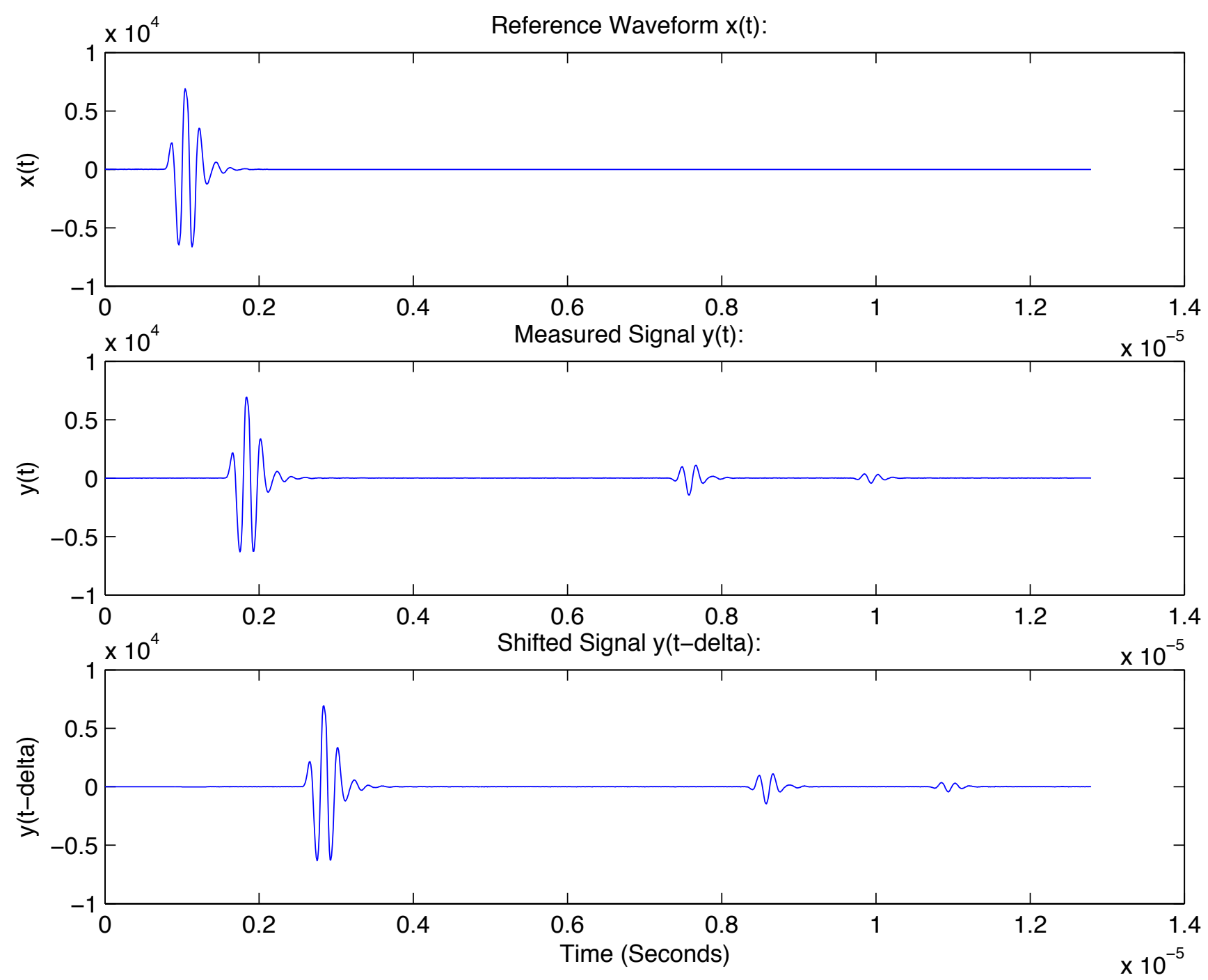

Figure 3.5: Reference signal $x(t)$, output measurement $y(t)$ and the delayed measurement $y(t-\Delta)$ plotted for comparison $(\Delta=100)$. 


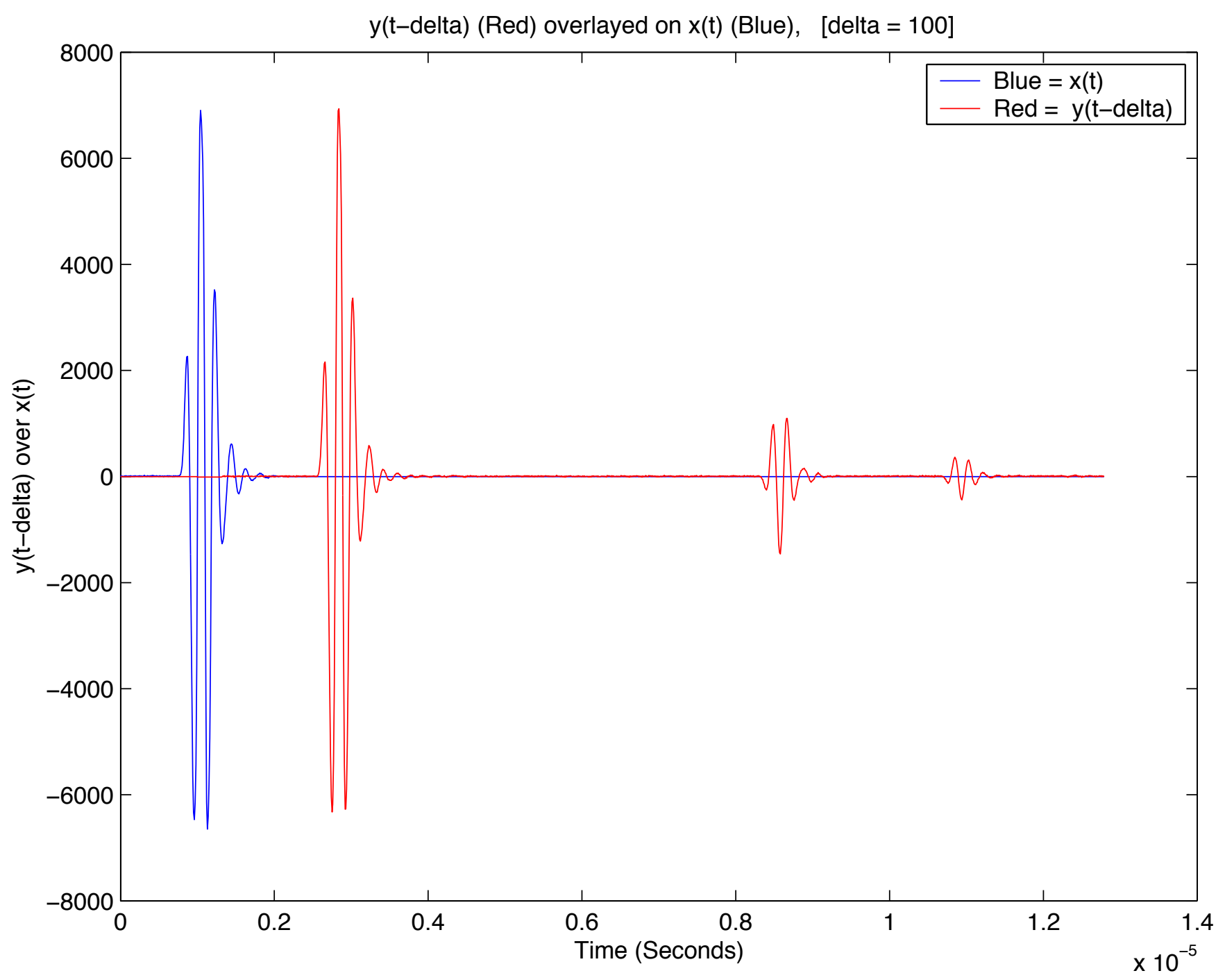

Figure 3.6: Reference $x(t)$, plotted over the delayed measurement $y(t-\Delta)$ for comparison. 
section), and applying bandlimited spectrum extrapolation algorithms as proposed at the end of this report and as described in [1].

An example of decimating the data is depicted in the following figures.

Figure (3.7) shows $|X(f)|^{2}$ and $|Y(f)|^{2}$ before decimation. The signals are clearly oversampled.

Figure (3.8) shows the signals $x(t)$ and $y(t)$ after decimation by the noninteger ratio of 13/2.

Figure (3.9) shows $|X(f)|^{2}$ and $|Y(f)|^{2}$ after decimation by 13/2. The high frequency spectral zeros have been effectively eliminated. 

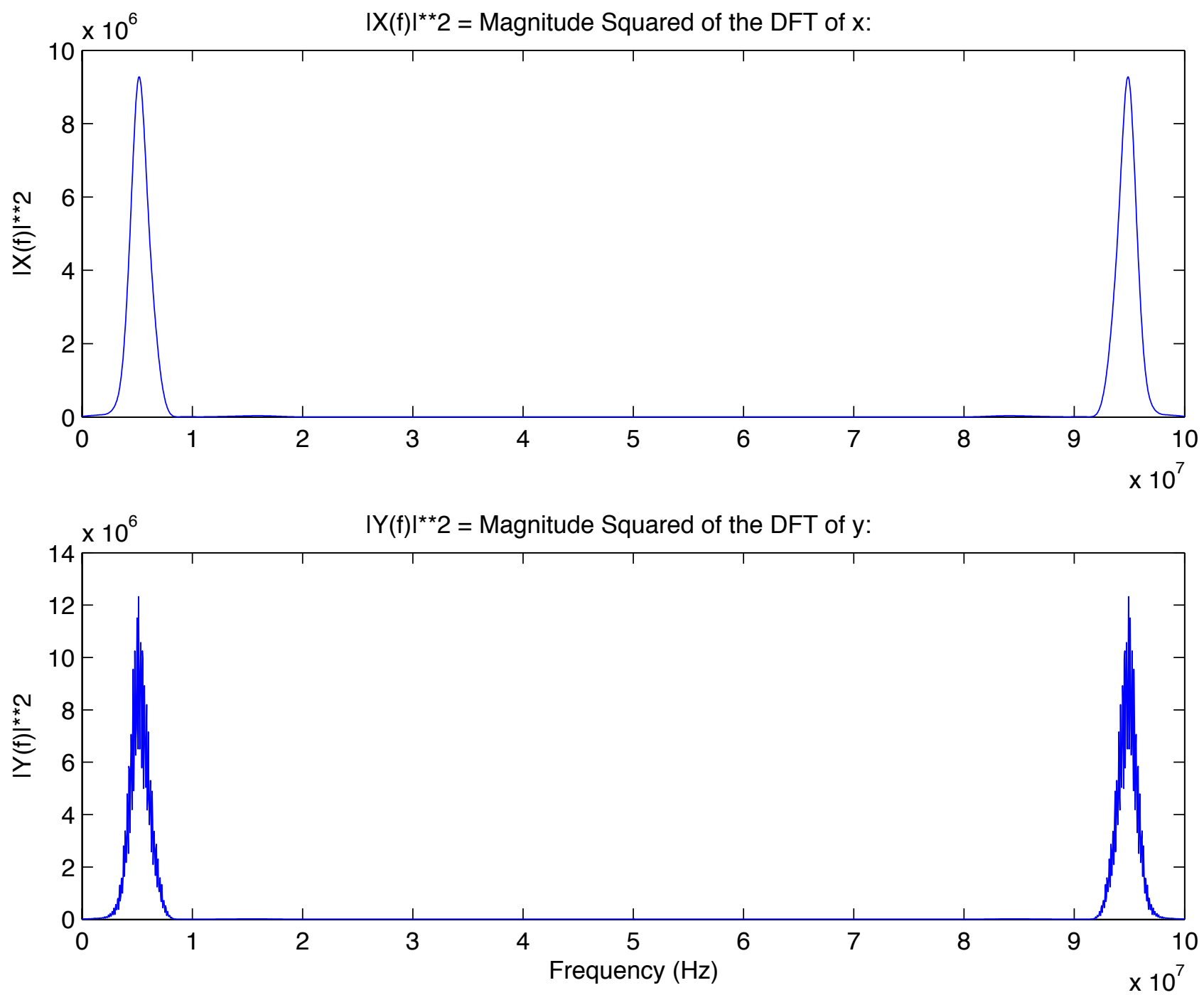

Figure 3.7: $|X(f)|^{2}$ and $|Y(f)|^{2}$ before decimation. The sampling frequency is $f_{s}=10 . e 7 \mathrm{~Hz}$, so the folding frequency is $f_{s} / 2=5 . e 7 \mathrm{~Hz}$. Note that due to oversampling, the number of spectral zeros is very large, making $R_{x x}$ ill-conditioned. 


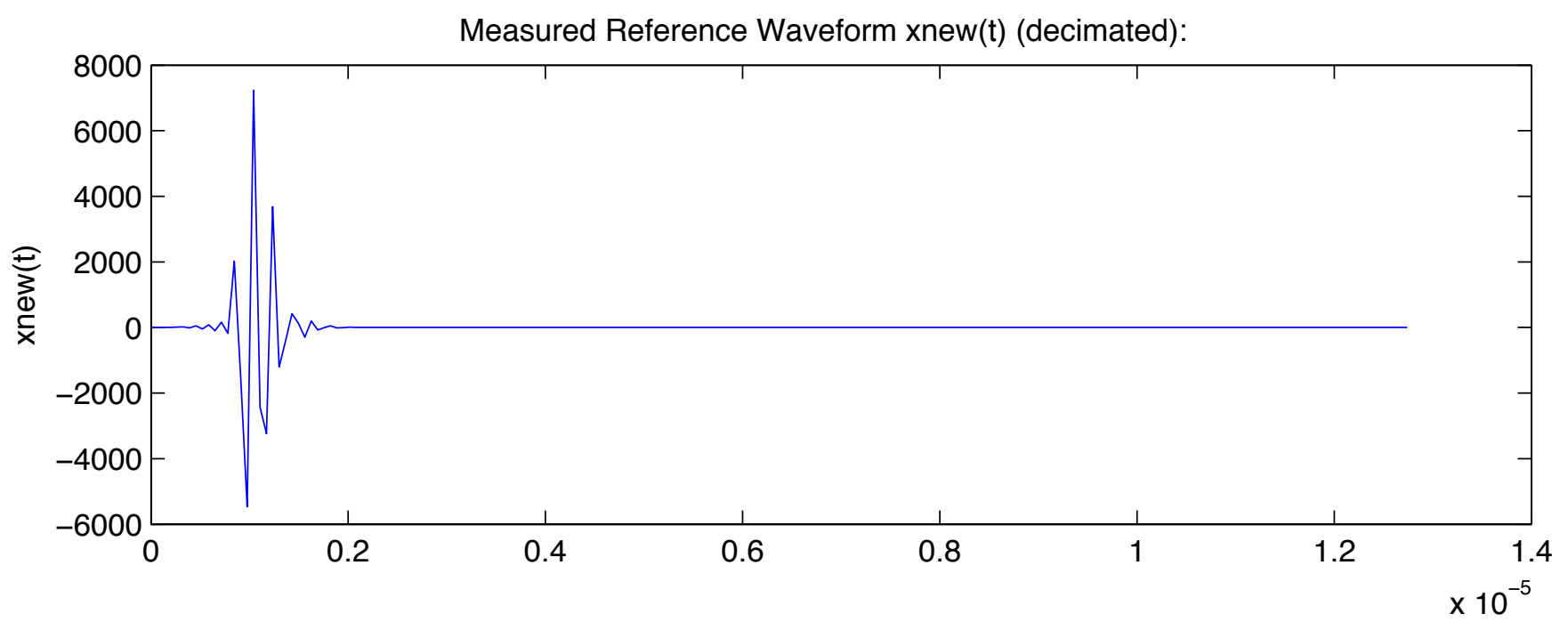

Measured Reflection Waveform ynew(t) (decimated):

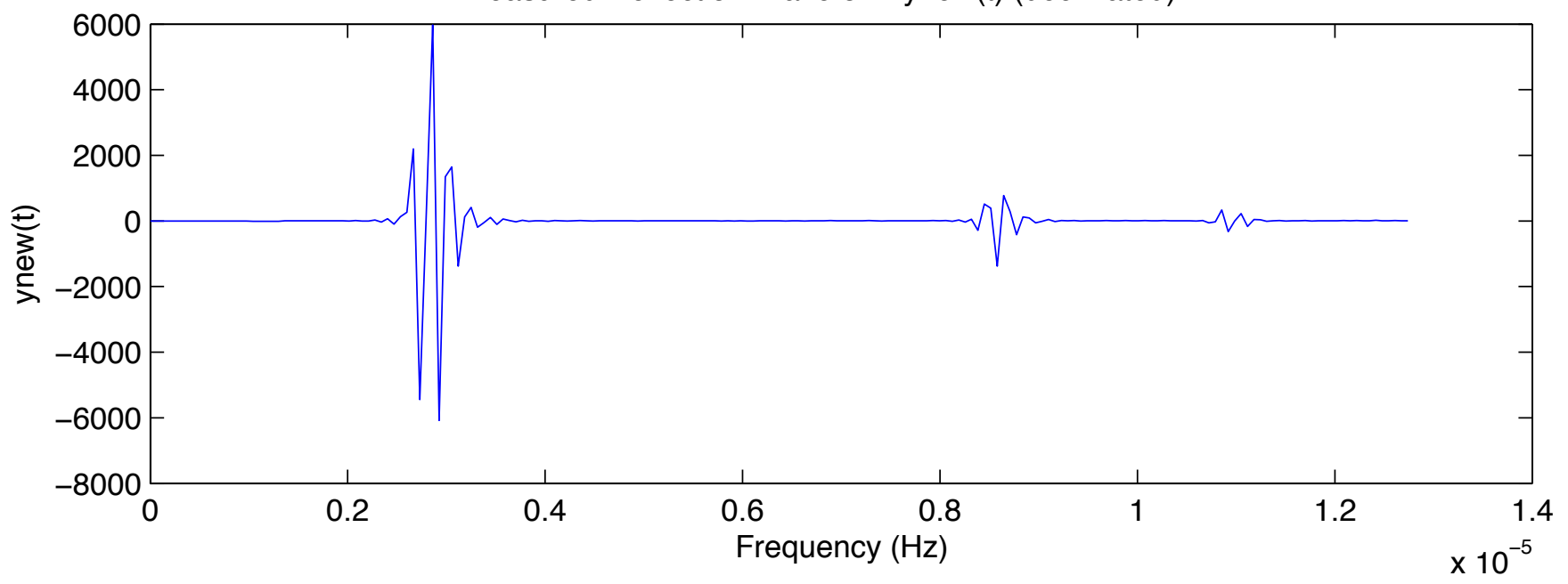

Figure 3.8: The signals $x(t)$ and $y(t)$ after decimation by $13 / 2$. 

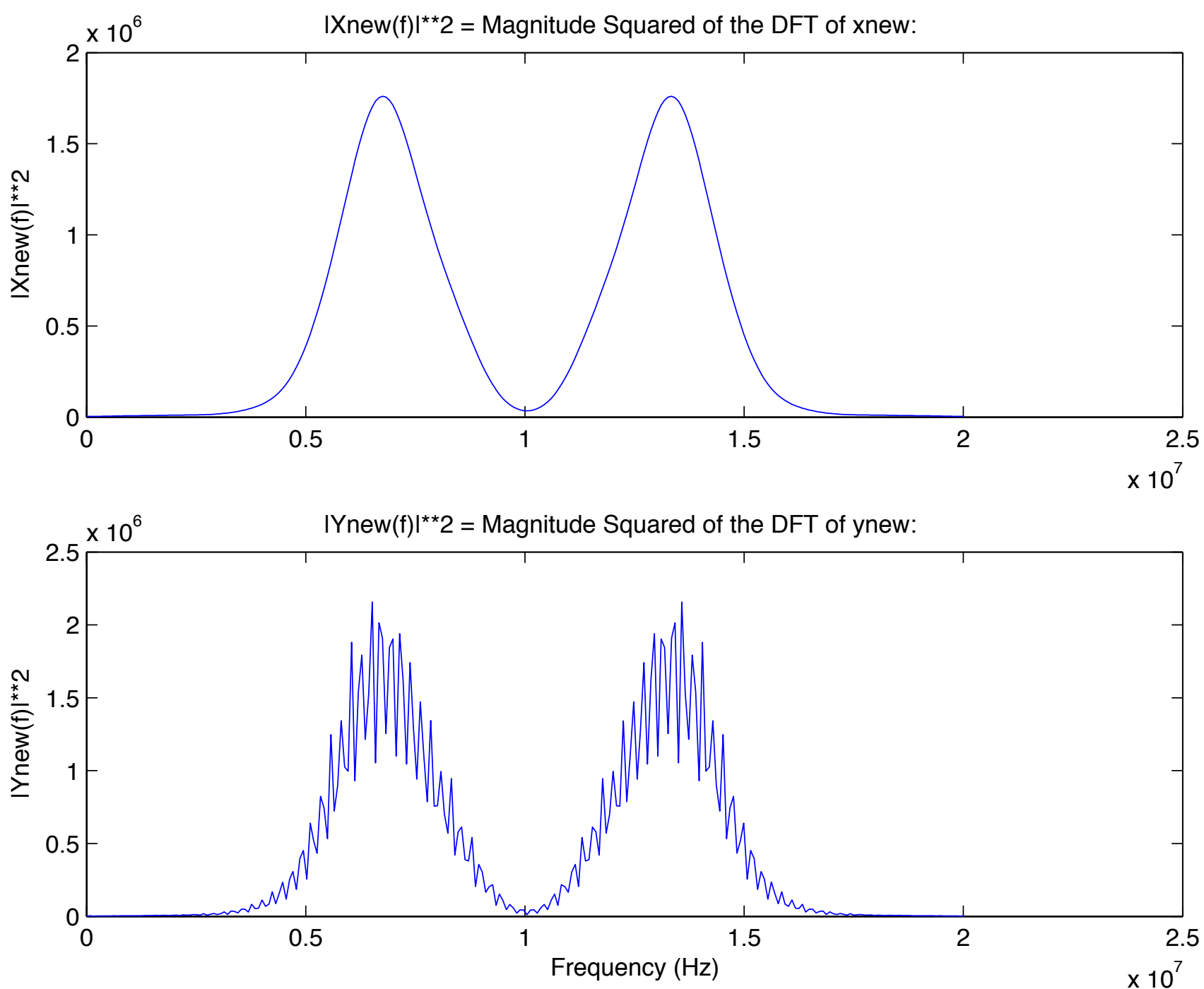

Figure 3.9: $|X(f)|^{2}$ and $|Y(f)|^{2}$ after decimation by 13/2. Note that the high frequency spectral zeros have been effectively eliminated by the decimation step. 


\section{Chapter 4}

\section{Impulse Response Estimation}

The MATLAB code for impulse response estimation is called "Wiener GAC". In this section, we apply the code to our example data and discuss the results.

Figure (4.1) shows the optimal least squares Wiener estimate of the impulse response $\widehat{h}(t)$. The polarity (phase) of the reflected pulses are now clearly evident to the author.

Figure (4.2) shows the estimated output $\widehat{y}(t)$ plotted over the measured output $y(t)$. The two signals match very closely. Also shown is the error signal $e(t)=y(t)-\widehat{y}(t)$. Notice that the magnitude scale for the error is very small, and that the error is very small, even in the regions of maximum error. $e(t)$.

Figure (4.3): The estimated output $\widehat{y}(t)$ is plotted along with the estimated impulse response $\widehat{h}(t)$ and the error

Figure (4.4): The input $x(t)$, output $y(t)$, estimated output $\widehat{y}(t)$ and the estimated impulse response $\widehat{h}(t)$ are plotted for comparison. 


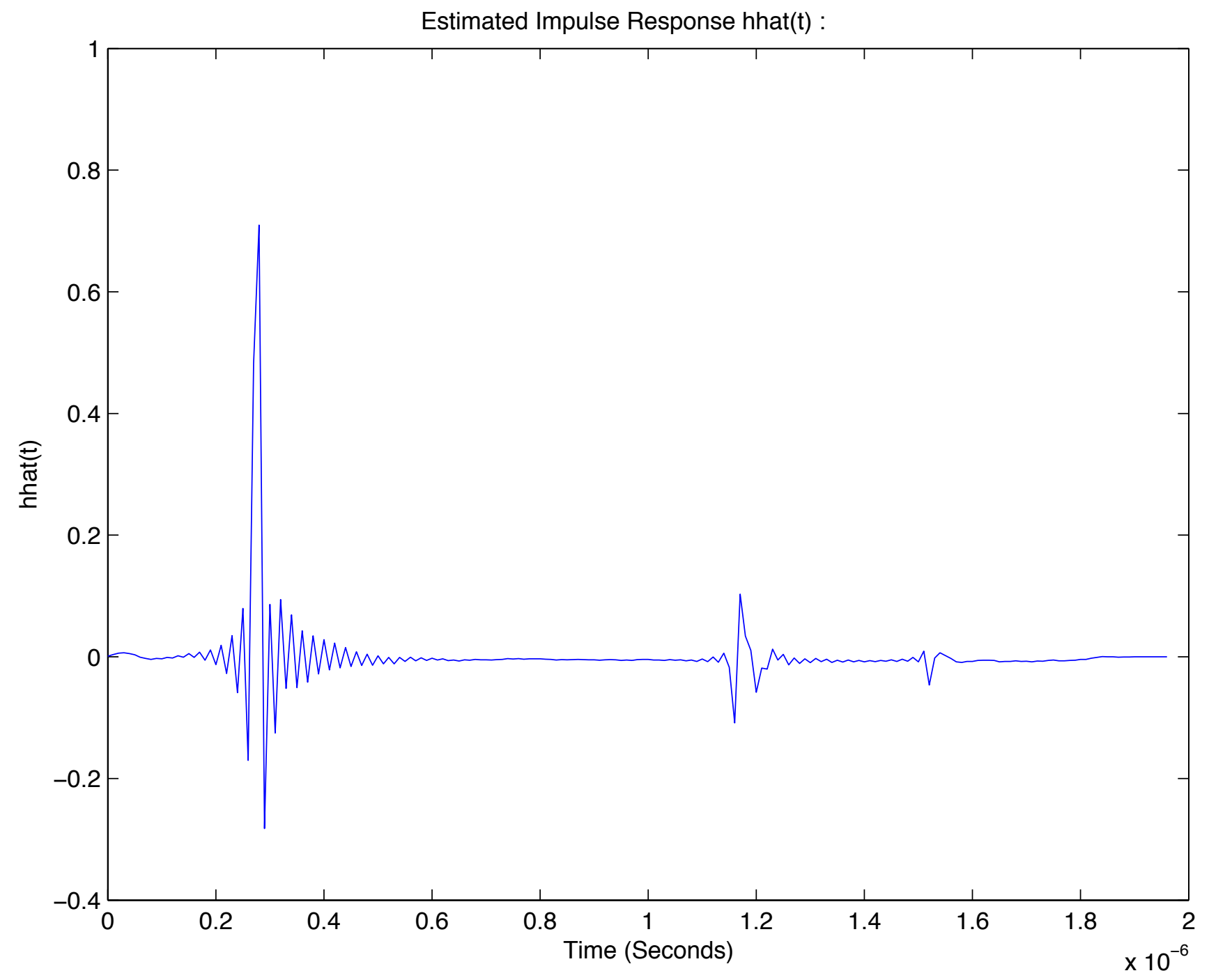

Figure 4.1: The optimal least squares Wiener estimate of the impulse response $\widehat{h}(t) . N=197, \alpha=5.0$, Range of $\alpha$ is .0182 to 8.7912. Note: we can now see clearly that, as predicted by theory, the polarity of the front reflection is positive, the polarity of the hole reflection is negative, and the polarity of the back reflection is negative. 

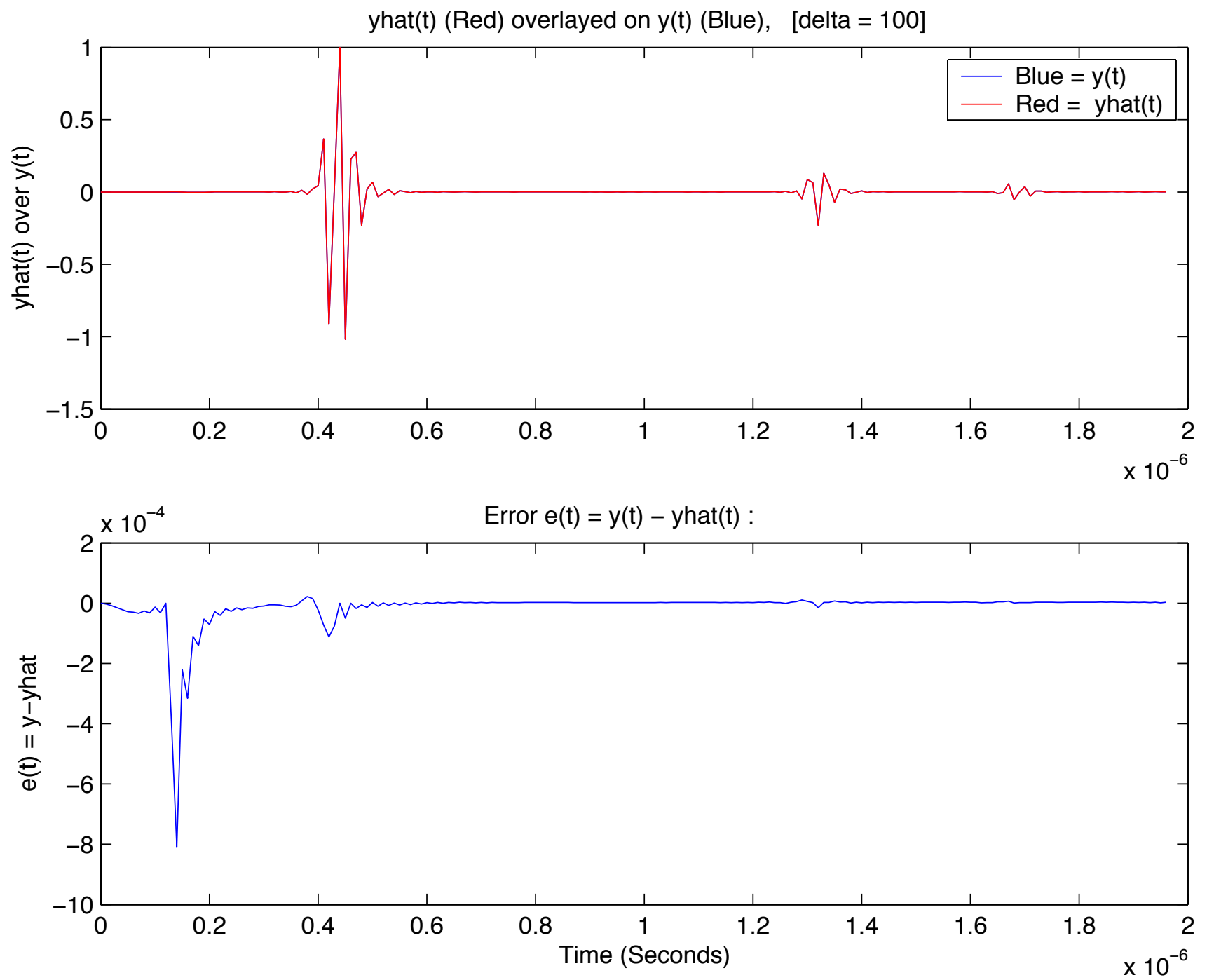

Figure 4.2: The estimated output $\widehat{y}(t)$ is plotted over the measured output $y(t)$. Also shown is the error signal $e(t)=y(t)-\widehat{y}(t)$. Notice that the magnitude scale for the error is very small, and that the error is very small, even in the regions of maximum error. 

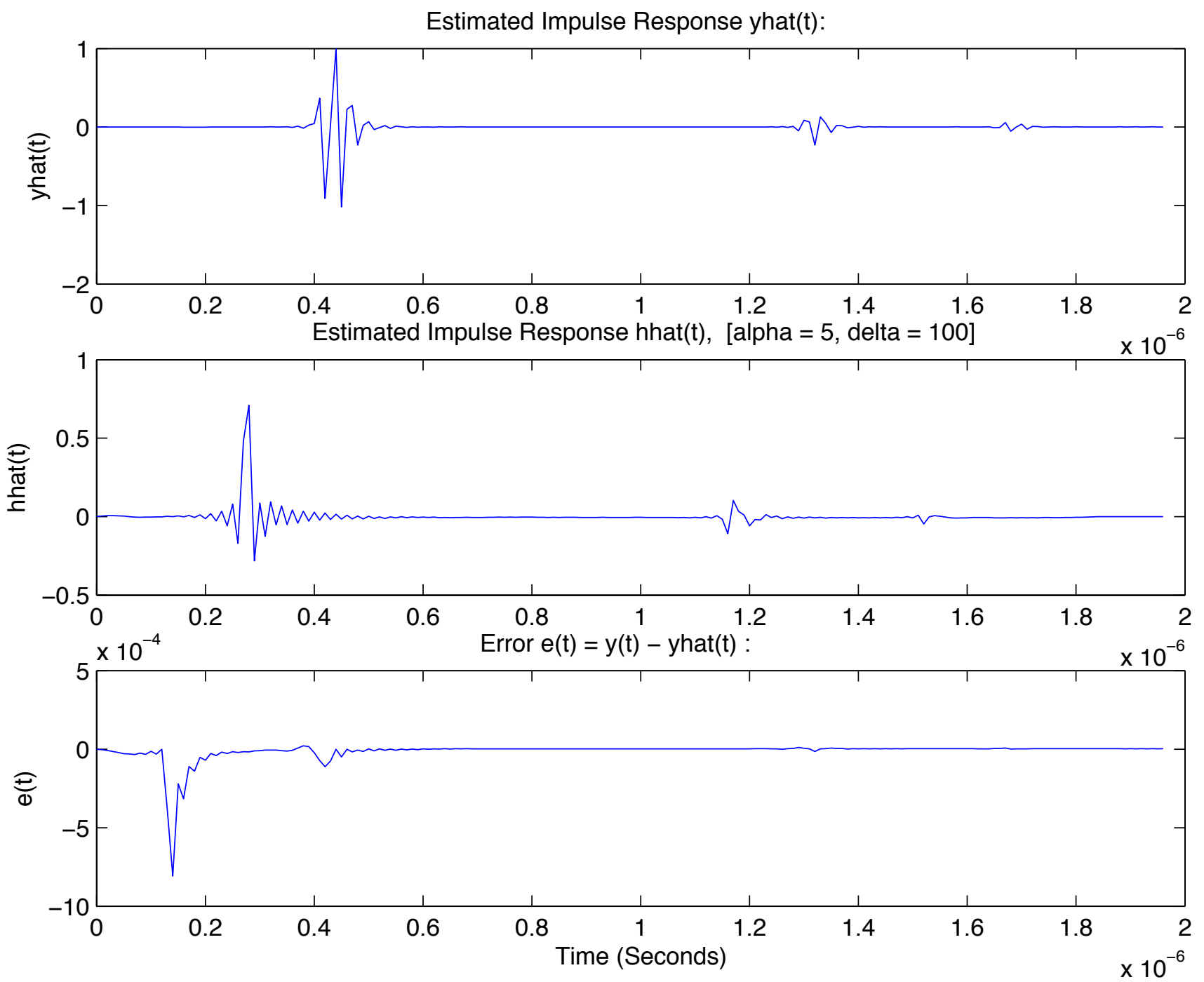

Figure 4.3: The estimated output $\widehat{y}(t)$ is plotted along with the estimated impulse response $\widehat{h}(t)$ and the error $e(t)$. 

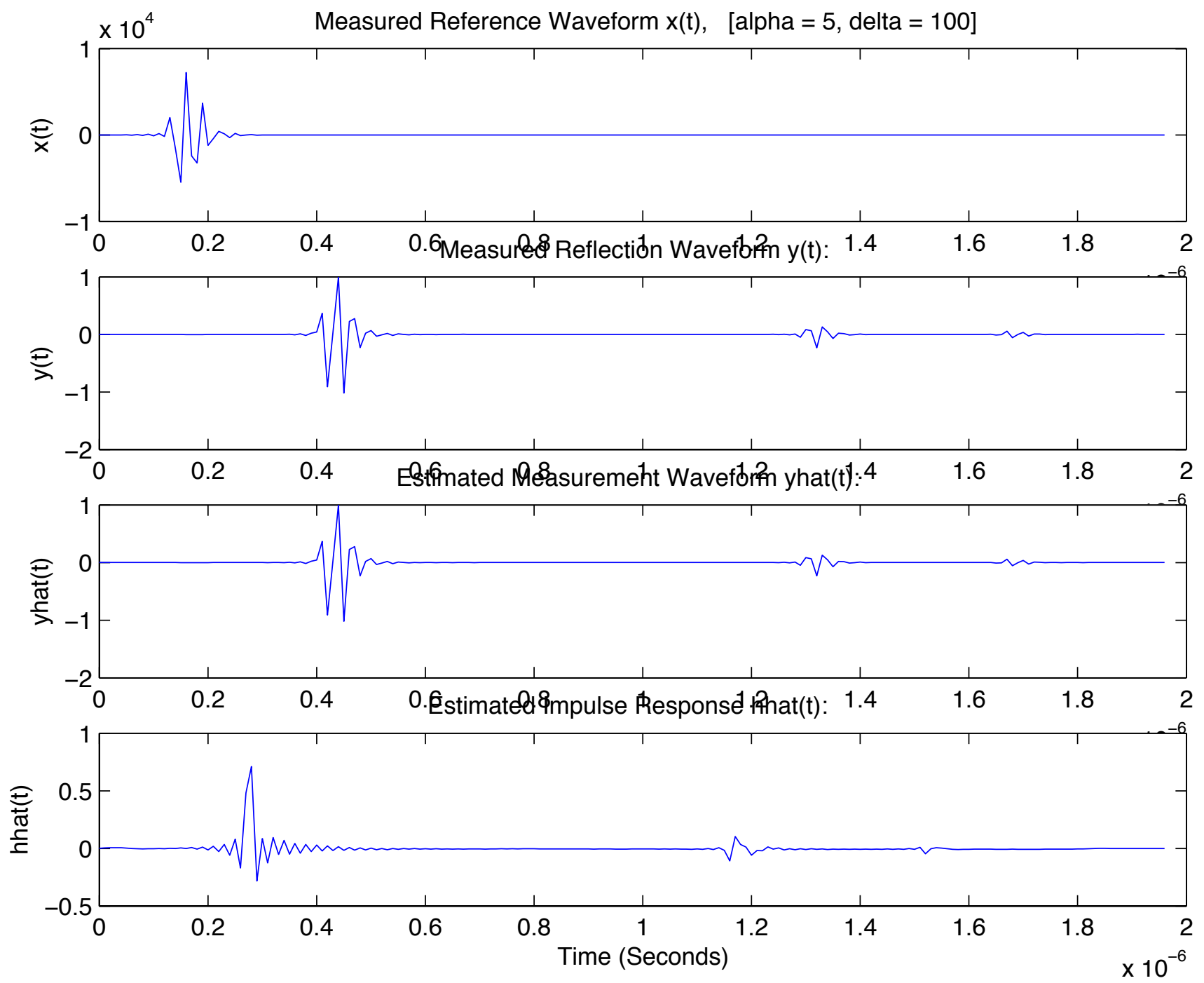

Figure 4.4: The input $x(t)$, output $y(t)$, estimated output $\widehat{y}(t)$ and the estimated impulse response $\widehat{h}(t)$ are plotted for comparison. Note that $\widehat{h}(t)$ does not align temporally with $y(t)$. 


\section{Chapter 5}

\section{Signal Post-Processing}

After the Wiener estimation algorithm is finished, some additional "post-processing " can be done to put the signals in a form for correct and easy interpretation.

\subsection{Including the Artificial Delay of $y(t)$ that was Introduced During Pre- processing}

Figure (5.1) The estimated measured output $y(t)$, the estimated impulse response $\widehat{h}(t)$ and the delayed estimated impulse response $\widehat{h}(t-\Delta)$ are plotted for comparison. Notice that once the artificial delay $\Delta$ introduced during preprocessing is included, the signals $y(t)$ and $\widehat{h}(t-\Delta)$ align nicely. This effect is more easily seen in the next figure.

Figure (5.2) The estimated delayed impulse response $\widehat{h}(t-\Delta)$ is plotted over the measured output $y(t)$ for comparison. Notice that once the delay $\Delta$ is included, the signals $y(t)$ and $\widehat{h}(t-\Delta)$ align nicely. This step is not absolutely necessary, but is may provide a display that is more pleasing to the eye.

\subsection{Interpolating (Up-Sampling) the Results}

This step is not necessary for many applications. In some cases, however, it may be advantageous to interpolate the estimation results to present a more pleasing display.

Figure (5.3) depicts the magnitude squared of the DFT's of $\widehat{y}(t)$ and $\widehat{h}(t),|\widehat{Y}(f)|^{2}$ and $|\widehat{H}(f)|^{2}$ before interpolation.

Figure (5.4) depicts $\widehat{y}(t)$ and $\widehat{h}(t)$ after interpolation by the noninteger ratio $2 / 13$.

Figure (5.5) depicts the magnitude squared of the DFT's of $\widehat{y}(t)$ and $\widehat{h}(t),|\widehat{Y}(f)|^{2}$ and $|\widehat{H}(f)|^{2}$ after interpolation by $2 / 13$..

Figure (5.6) depicts $\widehat{h}(t)$ and $\widehat{y}(t)$ after post-processing, including interpolation. 

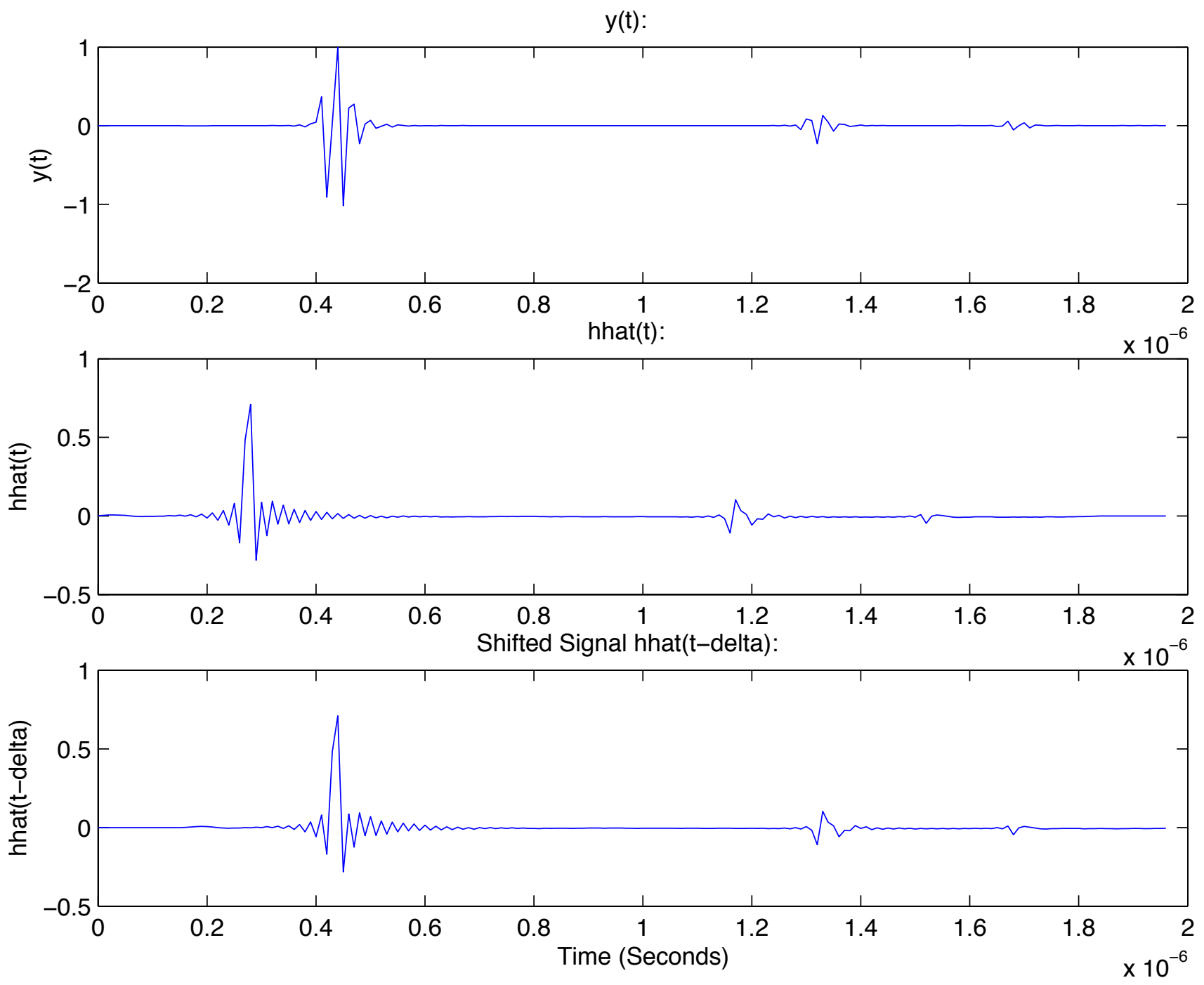

Figure 5.1: The estimated measured output $y(t)$, the estimated impulse response $\widehat{h}(t)$ and the delayed estimated impulse response $\widehat{h}(t-\Delta)$ are plotted for comparison. Notice that once the delay $\Delta$ is included, the signals $y(t)$ and $\widehat{h}(t-\Delta)$ align nicely. This effect is seen more easily in the next figure. 


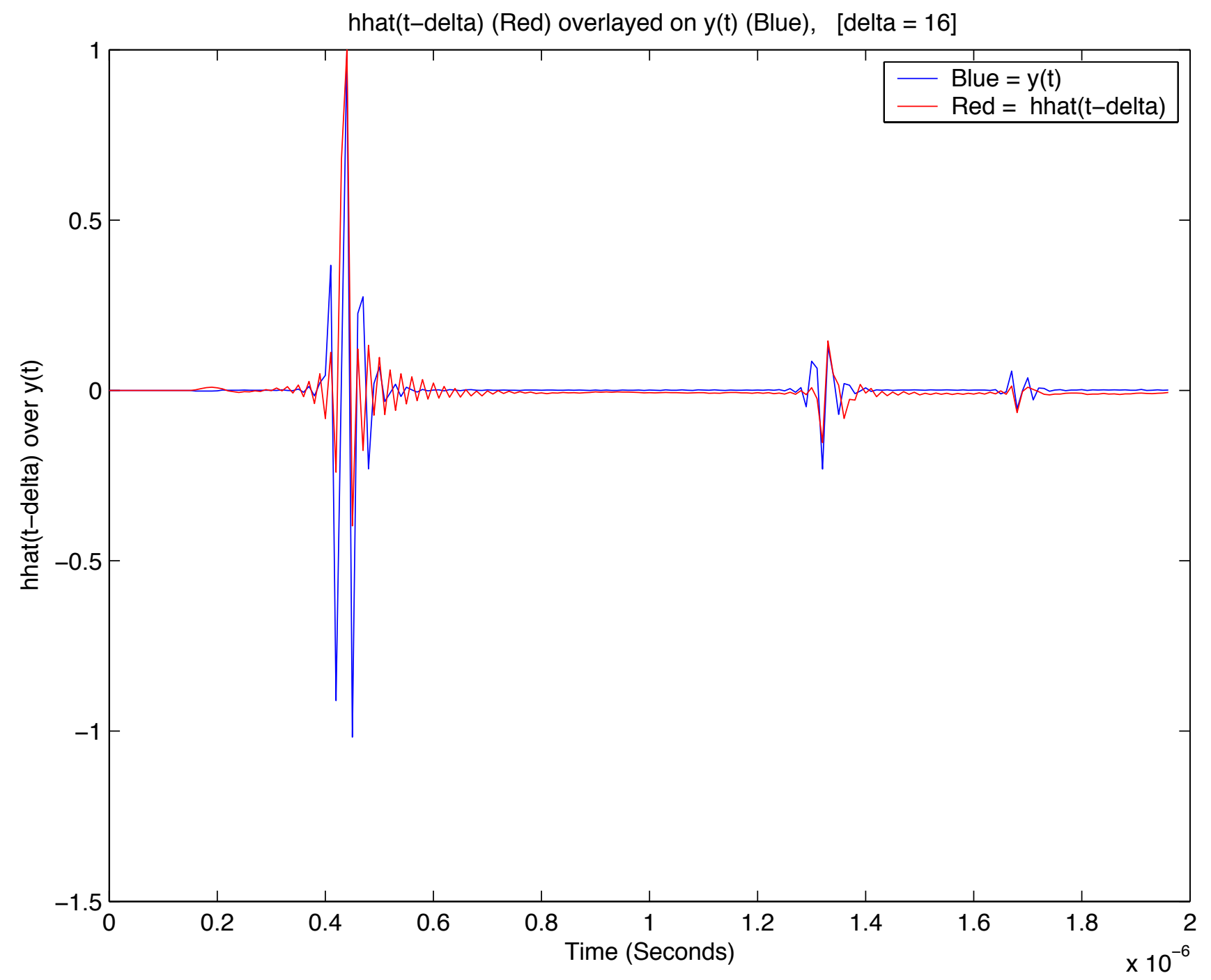

Figure 5.2: The estimated delayed impulse response $\widehat{h}(t-\Delta)$ is plotted over the measured output $y(t)$ for comparison. Notice that once the delay $\Delta$ is included, the signals $y(t)$ and $\widehat{h}(t-\Delta)$ align nicely. 


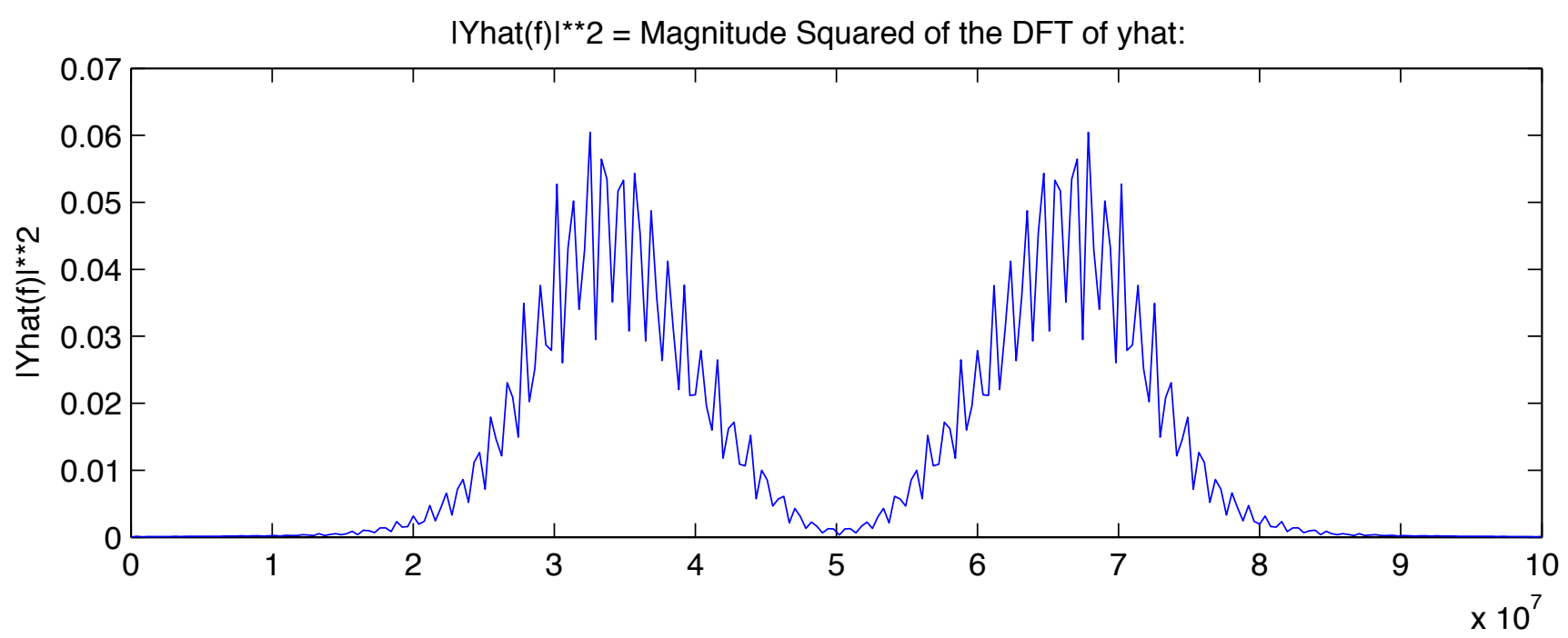

IHhat(f) $\left.\right|^{\star *} 2=$ Magnitude Squared of the DFT of hhat shifted:

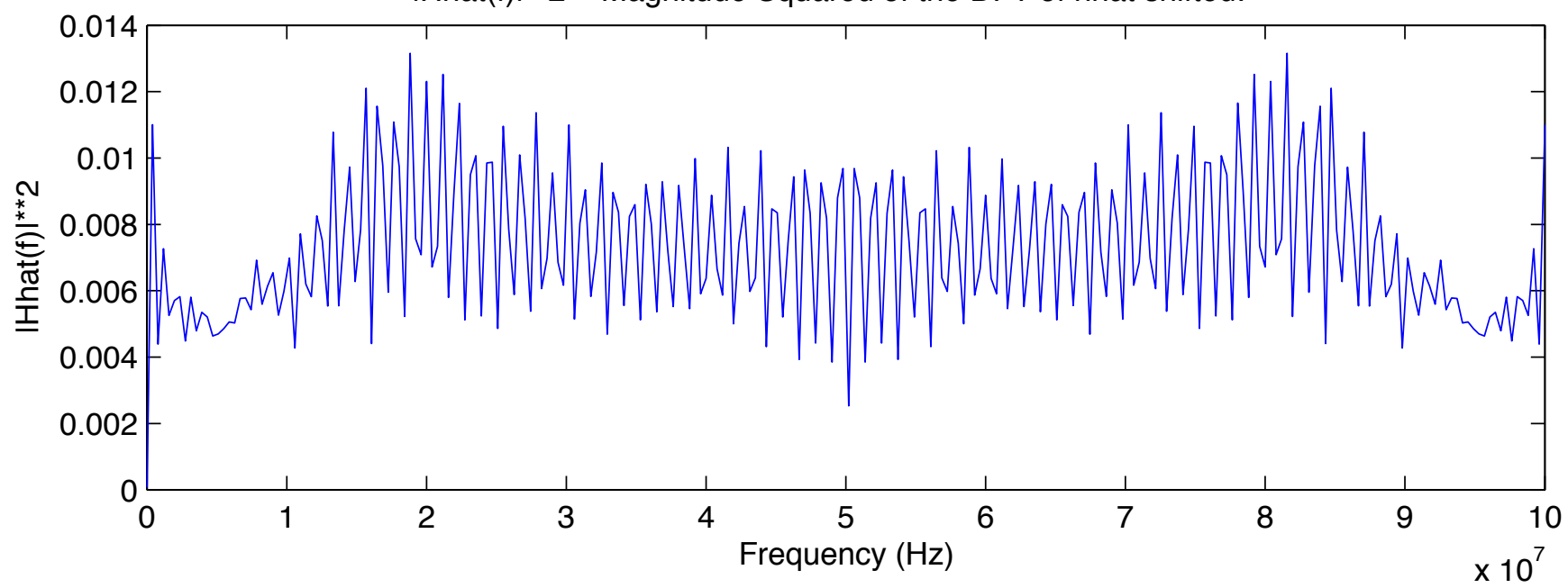

Figure 5.3: The magnitude squared of the DFT's of $\widehat{y}(t)$ and $\widehat{h}(t),|\widehat{Y}(f)|^{2}$ and $|\widehat{H}(f)|^{2}$ before interpolation. 

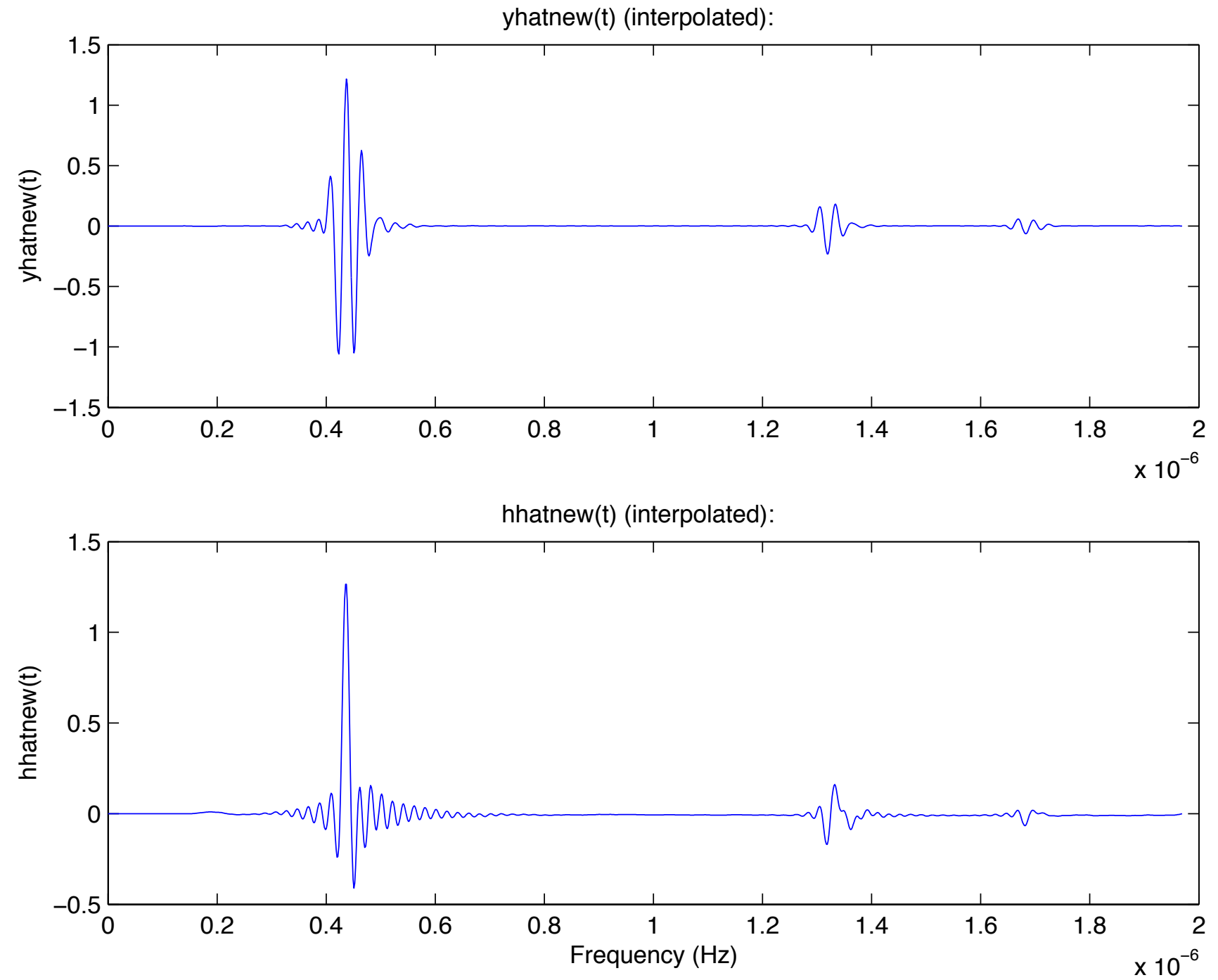

Figure 5.4: $\widehat{y}(t)$ and $\widehat{h}(t)$ after interpolation. For some users, this display may be more pleasing to the eye than the decimated version shown earlier. 


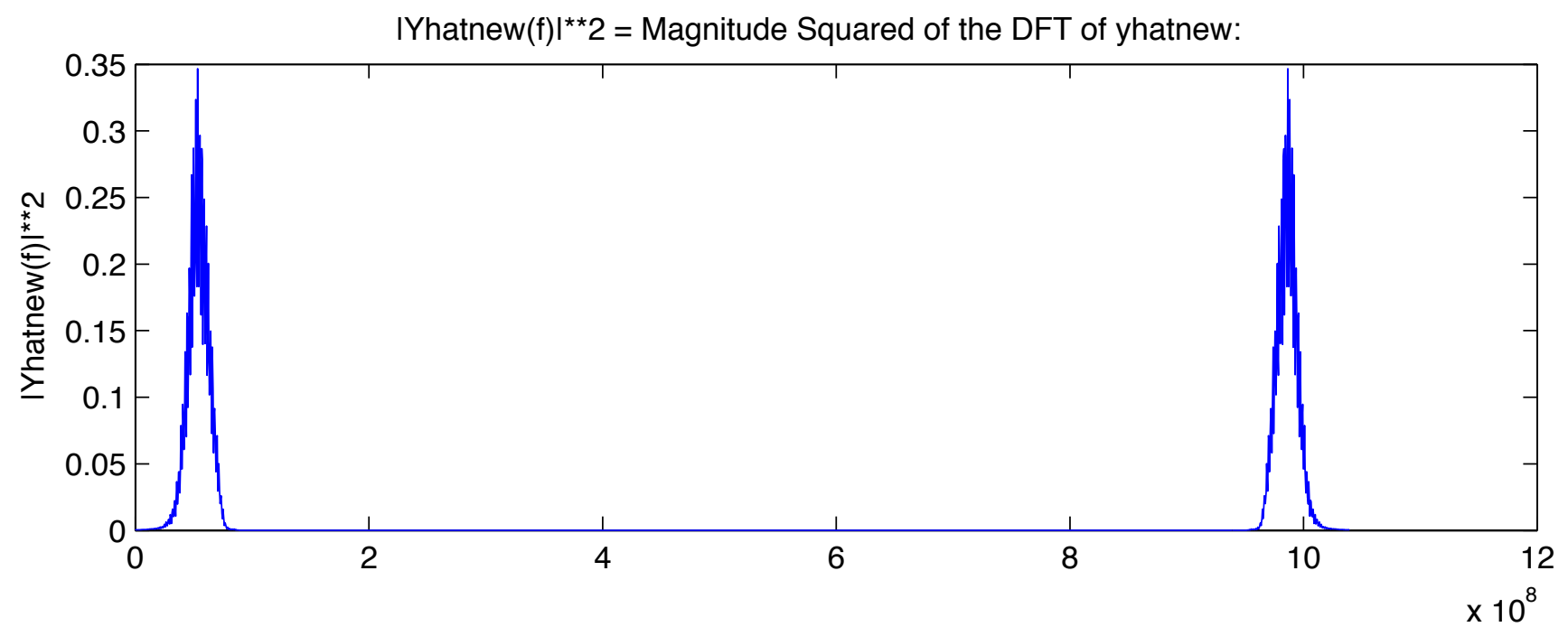

|Hhatnew $\left.(f)\right|^{* *} 2=$ Magnitude Squared of the DFT of hhatnew:

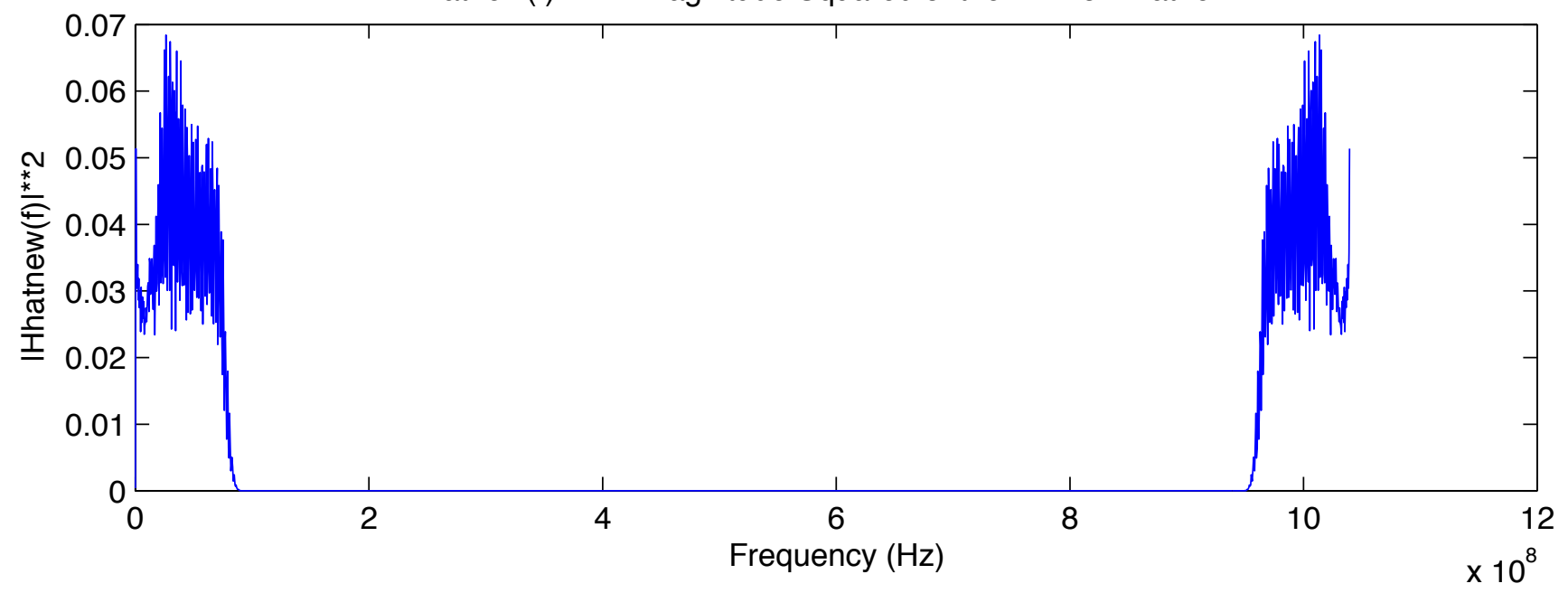

Figure 5.5: The magnitude squared of the DFT's of $\widehat{y}(t)$ and $\widehat{h}(t),|\widehat{Y}(f)|^{2}$ and $|\widehat{H}(f)|^{2}$ after interpolation by 2/13. 


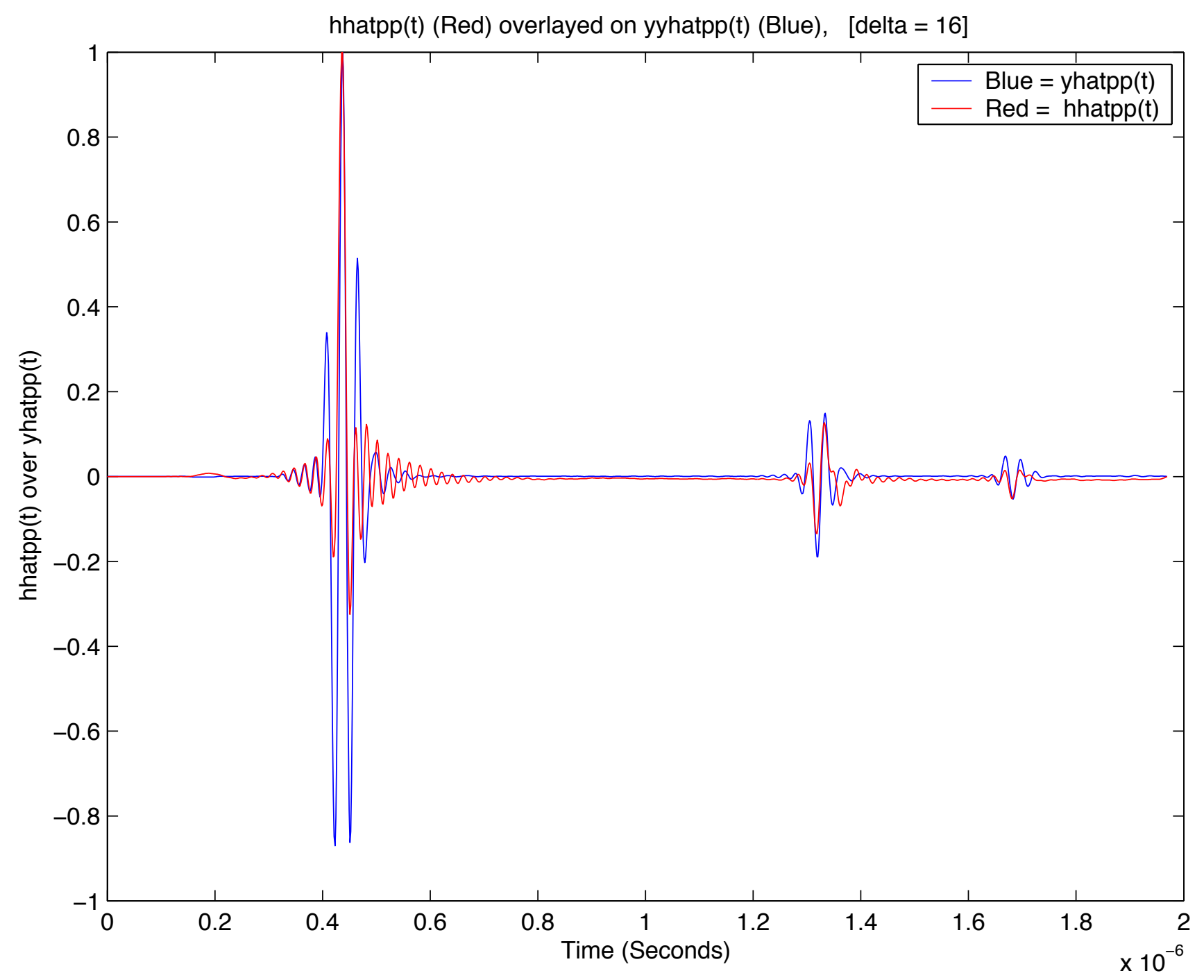

Figure 5.6: $\widehat{h}(t)$ plotted over $\widehat{y}(t)$ after post-processing, including interpolation. 


\section{Chapter 6}

\section{Processing Example Using a Back Surface Reflection as the Reference/Input Signal}

In the previous chapters, we demonstrated the signal processing algorithms using actual experimental signals measured in the laboratory. As a reference signal $x(n)$, we used the front surface reflection from the part under test. In most experimental situations, this is the most reasonable choice for a reference. The impulse response estimation results were satisfactory, but we are interested in exploring the possibility that better results might be achieved if we use a different, perhaps more appropriate reference signal. Perhaps it is beneficial to optimize the choice of reference signal to the hole reflection signal. For this problem, it makes sense to use the back surface reflection signal as a reference for the following reason: The back surface reflection is more likely to have the properties of the layer boundary. This is because the back surface reflection and the layer surface reflection have similar travel paths. The front surface reflection does not travel through any of the materials.

Note that if our goal is to sharpen the shape of the hole reflection wavelet, we must invert the sign of the back wall reference signal. This is because from theory, we know that the hole reflection should be a negative-going impulse (see the previous section). The back wall reflection is already negative, so inverting its sign in the reference signal will ensure that proper sign is given to the estimated impulse response at the hole reflection position.

In this chapter, we briefly show the results of cutting out the back surface reflection to use as a reference, and estimating the impulse response of the material using this new reference signal. We show a subset of the kinds of figures shown in the last section. The figures are self-explanatory.

We see that the use of the back wall reflection provides the advantage that it makes the pulse or wavelet corresponding to the hole reflection to have a shape closer to that of an ideal impulse. Thus, the back wall reflection may be a good choice as a reference signal for some applications.

As explained in the figure captions, we see a demonstration of the issues discussed in the theory section. We see the bias caused by the regularization of the estimation problem, and the associated bias vs. estimation error variance tradeoff. The value of the regularization parameter $\alpha$ was chosen to give minimum bias while maintaining acceptably small error variance. Further processing is possible to attempt to mitigate the low frequency bias; eg. highpass filtering. 


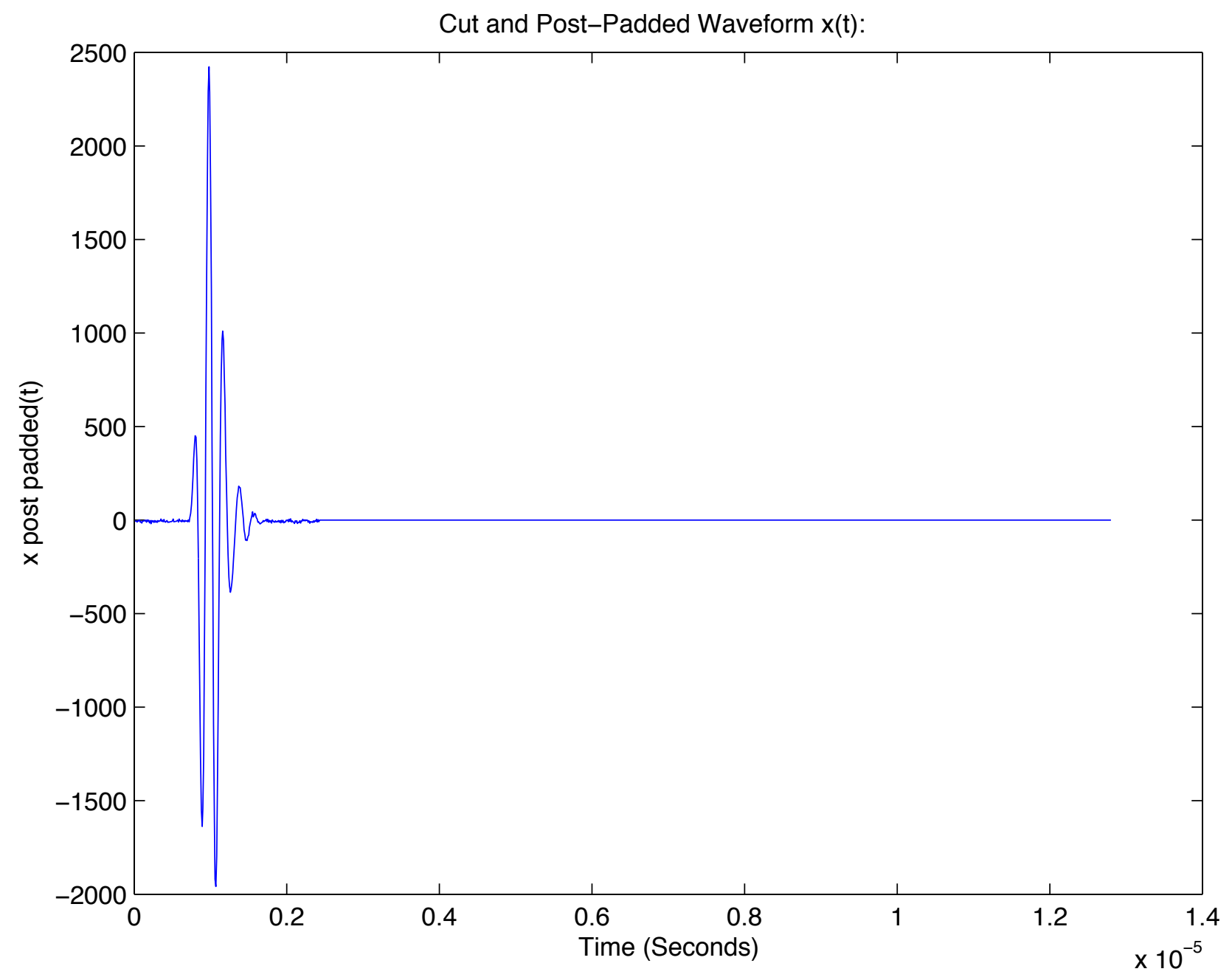

Figure 6.1: The cut and post-padded back surface reference signal $x(t)$. Note that the sign of the original back surface wavelet has been inverted in the reference signal. 

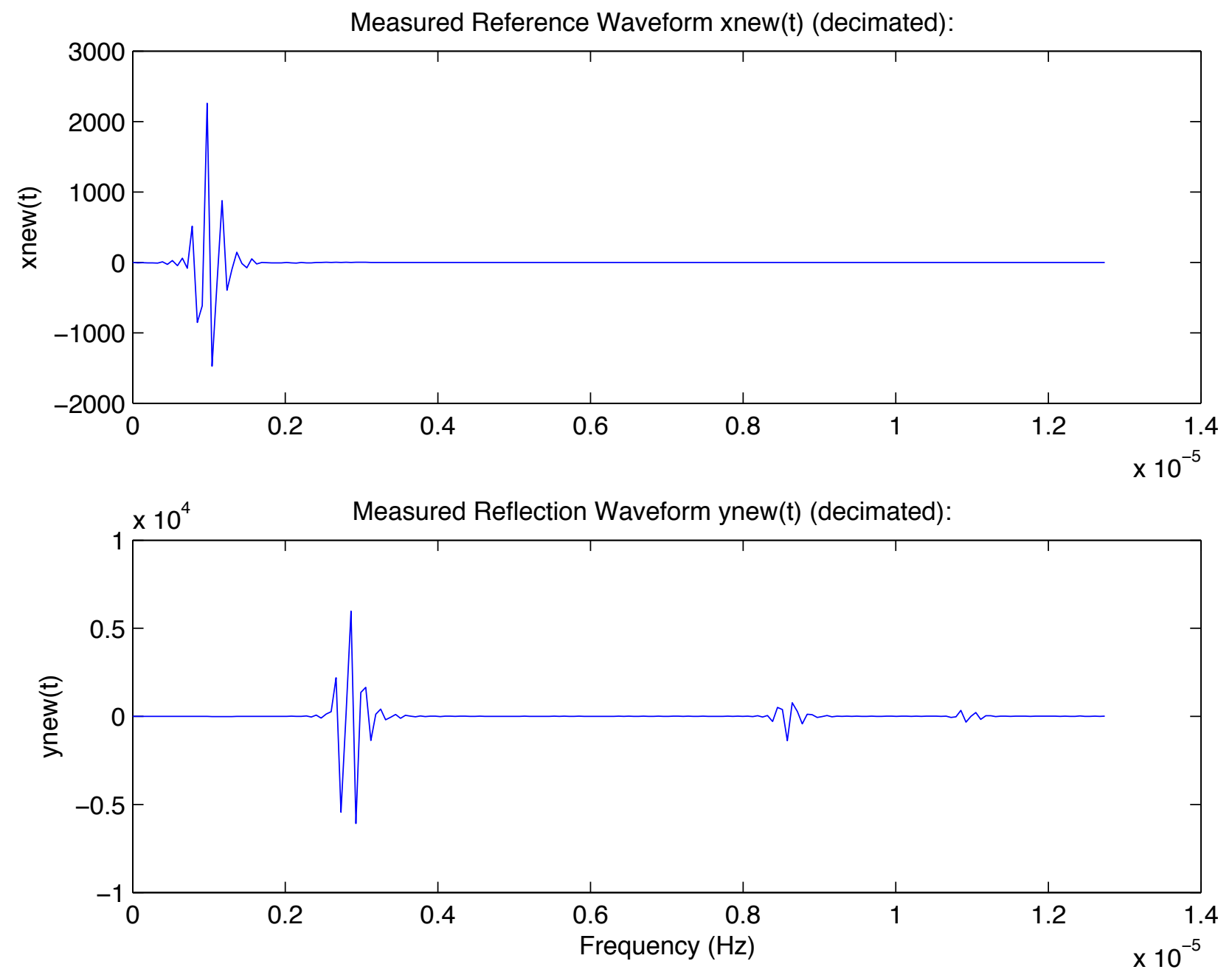

Figure 6.2: Back Surface Reference Case - The signals $x(t)$ and $y(t)$ after decimation by 13/2 

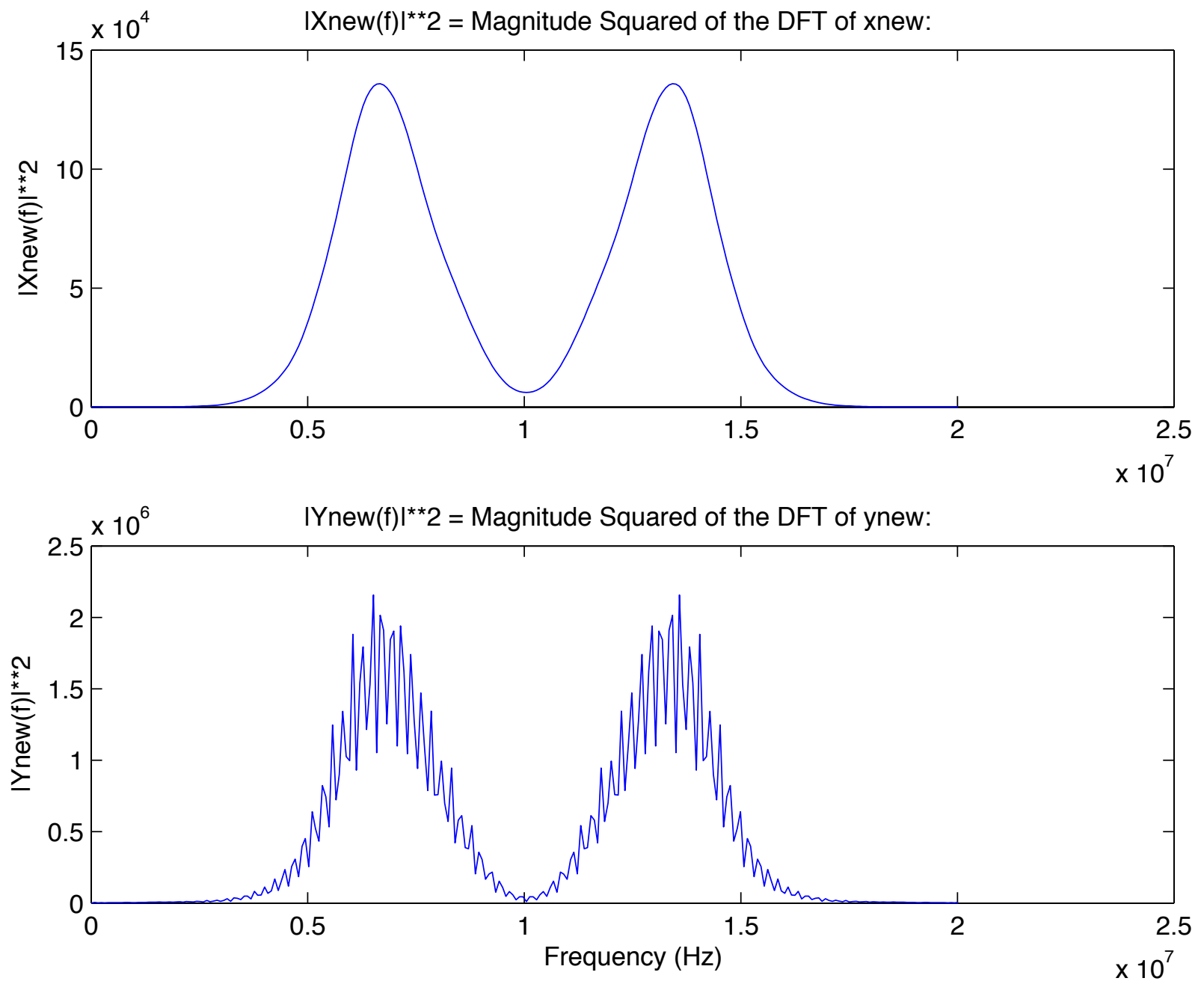

Figure 6.3: Back Surface Reference Case - $|X(f)|^{2}$ and $|Y(f)|^{2}$ after decimation by 13/2. Note that the high frequency spectral zeros have been effectively eliminated by the decimation step. 


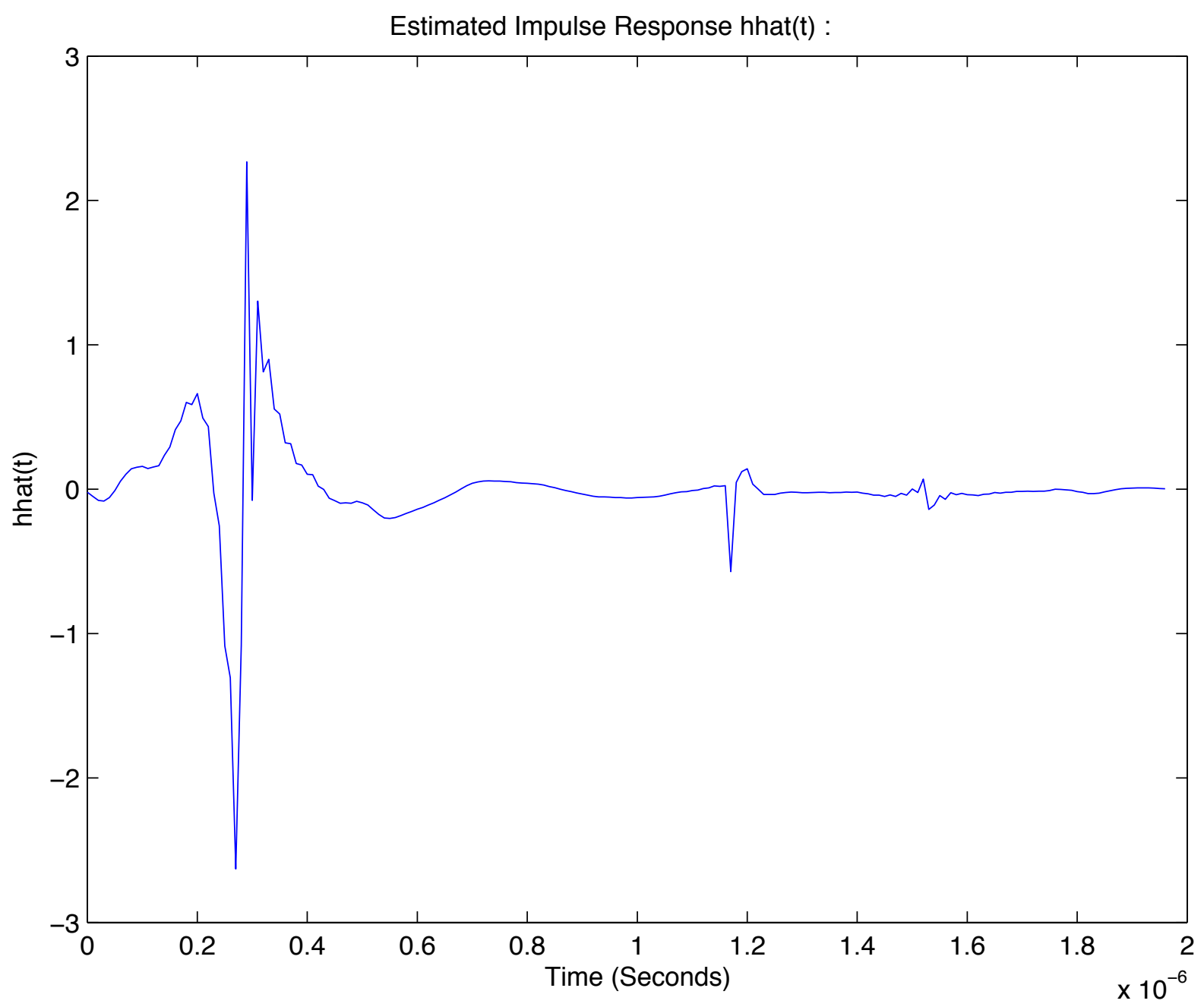

Figure 6.4: Back Surface Reference Case - The optimal least squares Wiener estimate of the impulse response $\widehat{h}(t) . N=197, \alpha=6.7885$, Range of $\alpha$ is .0001 to 6.7885 . Note: we see that the front reflection is more distorted relative to the case in which we used a front surface reflection as the reference signal. This is, of course, expected, because the back reflection does not match the front reflection. The polarity of the hole reflection is now clearly negative, as we expect from theory. In addition, the hole reflection wavelet now has more of a "spike" "shape as desired. However, the bias in the estimate caused by the regularization is apparent in the low frequency trend introduced into the signal. Fortunately, the bias is probably not enough to be detrimental for our purposes. Also, further post-processing can mitigate it. 


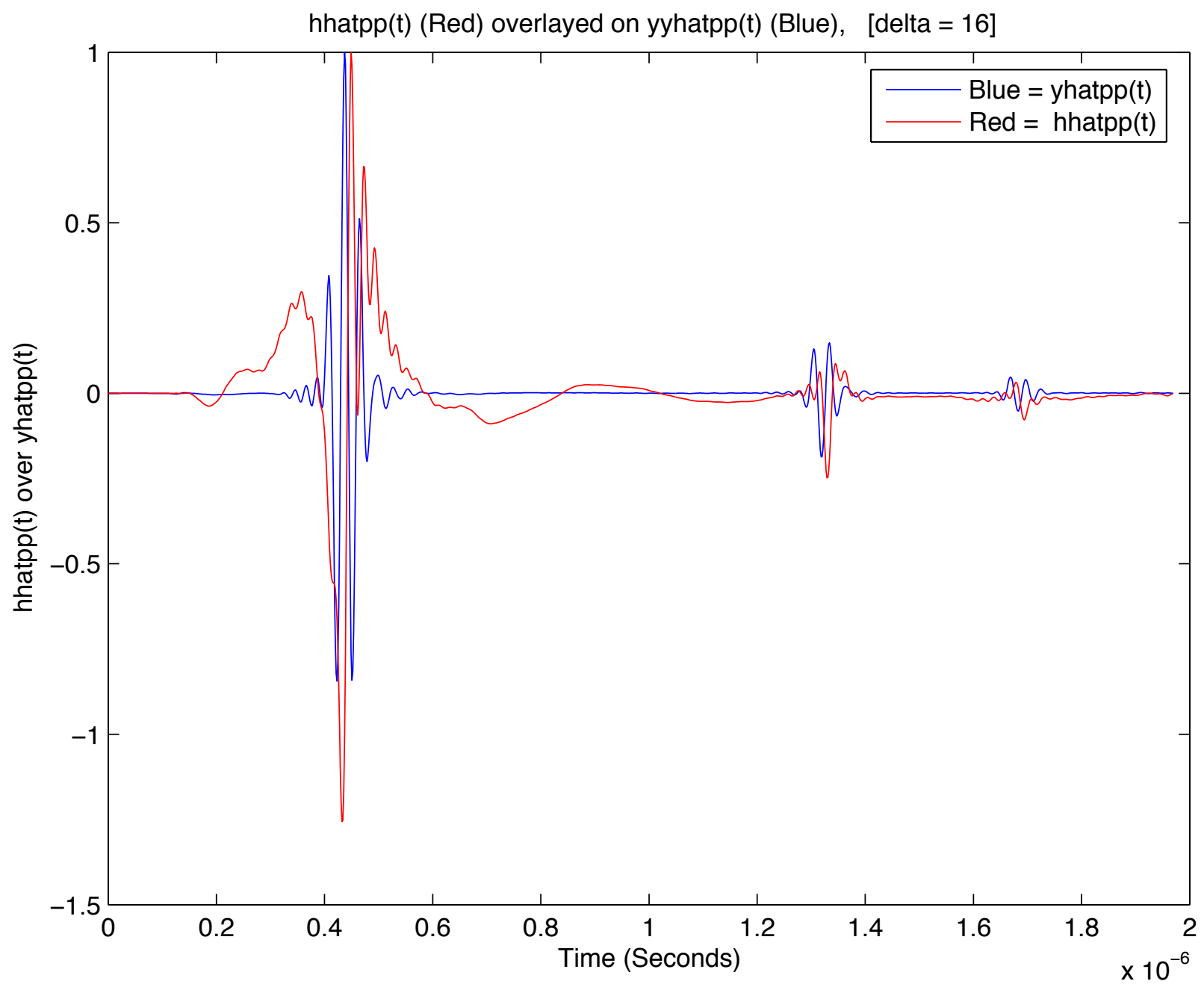

Figure 6.5: $\widehat{h}(t)$ plotted over $\widehat{y}(t)$ after post-processing, including interpolation. The bias in the estimate caused by regularization is apparent in the low frequency trend introduced into $\widehat{y}(t)$. Fortunately, the bias is probably not enough to be detrimental for our purposes. Also, further post-processing can mitigate it. 


\section{Chapter 7}

\section{Future Work}

The next step in our current work is to rewrite this research code to create a production code for use with large data sets, including B-scans and blocks of B-scans.

It is recommended that future projects allow for implementing an algorithm for bandlimited spectrum extrapolation [1]. This allows us to expand artificially the bandwidth of the transducers using analytic continuation from complex variables theory [32]. The author has used the method of alternating orthogonal projections very effectively for ultrasonic NDE [1]. Following the Wiener impulse response estimation with bandlimited spectrum extrapolation provides us with much sharper impulses in the estimated impulse response at material boundaries. Spatial resolution is, therefore, greatly enhanced [1].

Some work is planned to highpass filter the estimated impulse response for the back wall reference case. It is hoped that the lowpass regularization bias will be mitigated by this step.

Another possible avenue of approach is to use the "spiking filter "strategy developed for seismic signals [17, 18]. In this approach, the desired response for the Wiener filter is an impulse, and the Wiener filter estimates the impulse response that will achieve it. The approach proposed in this paper is the "shaping filter "approach, in which the desired response for the Wiener filter is the measured waveform. Generally, if a reference signal is available, the shaping filter approach is preferred because more information is available. However, for some applications, in which a good reference is not available, the spiking filter approach is very beneficial. 


\section{Chapter 8}

\section{Conclusions}

We have presented a brief discussion of a signal processing algorithm and MATLAB software for improving spatial resolution in ultrasonic nondestructive evaluation (NDE) imaging of materials. Given a measured reflection signal and an associated reference signal, the algorithm produces an optimal least-squares estimate of the impulse response of the material under test. We examined the use of two different reference signals, the front wall reflection and the back wall reflection. The estimated impulse response, when used in place of the raw reflection signal, enhances the spatial resolution of the ultrasonic measurements by removing distortion caused by the limited-bandwidth transducers and the materials under test. Two processing examples using actual ultrasonic measurements from a controlled experiment were provided as a tutorial. Even though the raw data have very high signal-to-noise ratio, the author is unable to identify the polarities (phase) of the two small reflections conclusively. From the estimated impulse response, however, the polarities are clear and correct. Proposed future work includes (1) Creating a production code for use with large data sets, including B-scans and blocks of B-scans, (2) Implementing and testing a bandlimited spectrum extrapolation algorithm for further enhancing the spatial resolution of the estimated impulse response, (3) Post processing the estimated impulse response to mitigate the regularization bias effects, and (4) Testing the spiking filter strategy often used when no appropriate reference signal is available. 


\section{Bibliography}

[1] G. A. Clark, D.M. Tilly, and W. D. Cook, Ultrasonic Signal/Image Restoration for Quantitative NDE, NDT International, Vol. 19, No. 3, June 1986.

[2] G. A. Clark and E. J. Bogdan, Summary of Rules for Sampling, Decimation and Interpolation of Experimental Data, Lawrence Livermore National Laboratory Report UCRL-19411, April 1982.

[3] J. V. Candy, G. A. Clark, and D. M. Goodman, Transient Electromagnetic Signal Processing: An Overview of Techniques, Chapter in the book, Time Domain Measurements in Electromagnetics, edited by Edmund K. Miller, Van Nostrand Reinhold Co., New York, 1986, pp. 416-455.

[4] G. A. Clark and F. L. Barnes, "Ultrasonic Signal Resolution Enhancement for Adhesive Thickness Measurements," Review of Progress in Quantitative NDE, Williamsburg, VA., June 21-28, 1987.

[5] G. A. Clark, S. E. Benson, and D. K. Lewis, Characterization of Wideband Ultrasonic Transducers Using Pulse Preshaping, Review of Progress in Quantitative NDE, Williamsburg, VA., June 21-28, 1987.

[6] G. A. Clark, Thresholds in Signal Processing, with Emphasis on Quantitative Nondestructive Evaluation of Materials, Invited paper, 1986 Precision Engineering Conference, American Society of Precision Engineering, Thresholds of Precision Engineering, Dallas, Texas, November 5-7, 1986.

[7] G. A. Clark and F. L. Barnes, Model-Based Parameter Estimation for Layer Problems in QNDE, Review of Progress in Quantitative NDE, U. of California San Diego, La Jolla, CA., Aug. 3-8, 1986.

[8] G. A. Clark, F. L. Barnes, and G. Thomas, Digital Signal and Image Restoration for Weld Underbead Profiling, Review of Progress in Quantitative NDE, U. of California San Diego, La Jolla, CA., Aug. 3-8, 1986.

[9] G. A. Clark, D. M. Tilly, B. D. Cook, J. M. Brase, Bandlimited Signal Extrapolation for A-and B-Scan Resolution Enhancement, Review of Progress in Quantitative NDE, Williamsburg, Virginia, June 23-28, 1985.

[10] G. A. Clark, J. M. Brase, B. D. Cook, D. M. Tilly, A-and B-Scan Resolution Enhancement Using System Identification Techniques, Review of Progress in Quantitative NDE, Williamsburg, Virginia, June 23-28, 1985.

[11] G. A. Clark, B. D. Cook, R. L. McKinney, G. D. Poe, Impulse Response of a Crack: Case Study of Signal Processing of Ultrasonic Signals, Symposium on Review of Progress in Quantitative NDE, U. of California, San Diego, July 8-13, 1984.

[12] B. D. Cook, G. A. Clark, R. L. McKinney, G. D. Poe, Impulse Response of a Crack: Case Study of Artifacts Produced by Transducer Misalignment, Symposium on Review of Progress in Quantitative NDE, U. of California, San Diego, July 8-13, 1984.

[13] B. D. Cook, G. A. Clark, and G. D. Poe, Application of Signal Extrapolation to Ramp Response Inverse Problems, Symposium on Review of Progress in Quantitative NDE, U. of California, San Diego, July 8-13, 1984. 
[14] D. Streit, G. A. Clark, and J. M. Brase, Computed Tomography for Quantitative NDE, Mechanical Engineering, September, 1987, pp. 56-64.

[15] G. A. Clark, Practical Aspects of Sampling and the Discrete Fourier Transform, LLNL Continuing Education Series, November 1983, with ongoing presentations over the past two decades.

[16] G. A. Clark, Elements of Digital Signal and Image Processing - Practical and Theoretical Preparation for MATLAB Training, Electronics Engineering Dept., Defense Sciences Engineering Division, Lawrence Livermore National Laboratory, UCRL-PRES-154699, September 2003.

[17] S. Treitel and E. A. Robinson, The Design of High-Resolution Digital Filters, IEEE Trans. Geoscience Electronics, Vol. GE-4, No. 1, 1966.

[18] E. A. Robinson and S. Treitel Geophysical Signal Analysis, Prentice Hall, 1980.

[19] R. M. Gray, On the Asymptotic Eigenvalue Distribution of Toeplitz Matrices, IEEE Trans. Information Theory, IT-18, pp. 725-730, 1972.

[20] J. V. Candy, Signal Processing: The Model-Based Approach, McGraw-Hill, New York, NY, 1986.

[21] J. V. Candy, Signal Processing: The Modern Approach, McGraw-Hill, New York, NY, 1988.

[22] H. W. Sorenson, Parameter Estimation, Marcel Decker, Inc., 1980.

[23] T. Kailath, Lectures on Linear Least-Squares Estimation, Spinger-Verlag, 1976.

[24] A. Papoulis, Probability, Random Variables and Stochastic Processes, McGraw-Hill, 1965.

[25] A. Jazwinski, Stochastic Processes and Filtering Theory, Academic Press, New York, NY, 1970.

[26] C. D. McGillem and G. R. Cooper,Continuous and Discrete Signal and System Analysis, Holt, Rinehart and Winston, 1974.

[27] A. V. Oppenheim, A. S. Willsky and I. T. Young, Signals and Systems, Prentice-Hall, H.J., 1983.

[28] R. E. Crochiere and L. R. Rabiner, Interpolation and Decimation of Digital Signals - A Tutorial Review, Proc. IEEE, Vol. 69, No. 3, MArch 1981.

[29] Digital Signal Processing Committee of ASSP, Programs for Digital Signal Processing, IEEE Acoustics, Speech and Signal Processing Society, IEEE Press, 1979.

[30] R. E. Crochiere and L. R. Rabiner, Multirate Digital Signal Processing, Prentice-Hall, 1983.

[31] B. Noble, Applied Linear Algebra, Prentice-Hall, 1969.

[32] R. V. Churchill Complex Variables and Applications, McGraw-Hill, 1960.

[33] MATLAB Reference Manual, The Mathworks, Natick Massachussetts, 1993. 